\title{
Il conflitto intrapsichico e l'evoluzione storica del suo approccio psicoterapeutico in psicoanalisi
}

\author{
Fulvio Frati*
}

SomMARIO. - Il concetto di 'conflitto intrapsichico' costituisce con ogni probabilità uno dei costrutti teorici sui quali la Psicoanalisi ha maggiormente accentrato la propria attenzione, dalla sua nascita sino ai giorni nostri, sia nel percorso di formazione e di modificazione nel tempo della propria struttura teorica complessiva che dal punto di vista della costruzione e del progressivo percorso di affinamento della propria tecnica psicoterapeutica. L'Autore, in questo articolo, cerca pertanto di presentare le principali tappe di questo lungo ed articolato percorso, sino a cercare di descrivere come il concetto di 'conflitto intrapsichico' potrebbe ormai considerarsi superato all'interno di uno tra i principali approcci psicoanalitici contemporanei, quello della Psicoanalisi della Relazione.

Parole chiave: Conflitto intrapsichico; conflitto relazionale; modello pulsionale; psicoanalisi della relazione; teoria dell'attaccamento.

\section{Introduzione}

Non esiste una precisa 'data di nascita' della Psicoanalisi: probabilmente la sua fase iniziale può comunque essere collocata all'incirca nel quinquennio 1890-1895, poiché - come afferma Brenner (1955, pag. 11) - nel 1895 l'evoluzione di questa disciplina appare 'già ben avviata'. Sono invece chiaramente condivisi dagli storici sia il nome del suo fondatore, Sigmund Freud (1856-1939), sia la caratteristica innovativa fondamentale che sin dall'inizio ha differenziato questa disciplina dalle altre a Lei contemporanee, vale a dire la sistematizzazione scientifica di un concetto in realtà a quel tempo già conosciuto e citato ma ancora scarsamente definito e descritto e, cioè, il concetto di 'inconscio'.

Sin dall'inizio, sostanzialmente, i principali 'capisaldi' della concettua-

*Psicologo, Psicoterapeuta e Psicoanalista SIPRe.

E-mail: fulviofrati@inwind.it 
lizzazione dell'Inconscio operata da questa nuova disciplina sono stati i seguenti: i) la 'psiche' non s'identifica con la coscienza; ii) la realtà psichica primaria non è 'il conscio' ma 'l'inconscio', in quanto il conscio rispetto all'inconscio è in realtà come 'la punta dell'iceberg' (vale a dire solo la manifestazione derivata e superficiale dell'inconscio); iii) nell'eziologia (cioè nello 'studio delle cause') delle malattie psichiche, un ruolo assolutamente fondamentale va sempre e comunque ricercato nel 'conflitto fra forze psichiche inconsce' (e quindi non necessariamente, o comunque non soltanto, nella presenza di disturbi organici).

\section{La struttura della psiche ('prima topica')}

Nei primi anni di sistematizzazione scientifica di questa nuova disciplina, Freud mise a punto un primo 'modello della psiche' (oggi conosciuto col nome di 'prima topica') che vedeva l'apparato psichico come suddiviso in tre specifiche 'zone', rispettivamente denominate - sulla base della fondamentale caratteristica di ciò che Freud riteneva che in ciascuna di esse fosse contenuto - come 'conscio', 'preconscio' e 'inconscio': i) conscio: sede di tutto ciò che è immediatamente accessibile ed utilizzabile nel funzionamento mentale di ciascuna persona; ii) preconscio: sede dei contenuti psichici che, pur essendo o comunque potendo apparire momentaneamente inconsci, possono in realtà divenire consci tramite uno sforzo non particolarmente eccessivo dell'attenzione; iii) inconscio: sede dei contenuti psichici stabilmente inconsci, che vengono mantenuti tali dalla forza di un meccanismo mentale denominato 'rimozione' la quale può essere superata soltanto attraverso tecniche apposite e specificatamente al riguardo ideate dalla psicoanalisi. Per tale ragione, il contenuto di questo 'luogo della mente' umana venne definito da Freud come 'il rimosso'.

\section{La rimozione nella 'prima topica'}

La rimozione, in questa fase dell'evoluzione della teoria freudiana, è quindi quel meccanismo di funzionamento psichico (o, per l'esattezza, uno dei vari 'meccanismi di difesa' che verranno in seguito ad uno ad uno descritti sia da Freud stesso sia da svariati altri psicoanalisti, in modo particolare da sua figlia Anna) il cui fine essenziale è quello di allontanare dalla coscienza quei desideri, pensieri o ricordi considerati dal soggetto inaccettabili ed insostenibili, e la cui presenza attiva nella sua mente cosciente gli provocherebbe invece dispiacere, dolore, sofferenza.

Già negli anni della 'prima topica', cioè praticamente dagli albori della psicoanalisi sino al 1923, Freud affianca inoltre alla sua visione 
della psiche umana come suddivisa nelle tre 'zone' una visione 'dinamica', secondo la quale queste tre 'aree' non sono semplicemente dei 'contenitori inerti' del materiale conscio, preconscio e Inconscio, ma sono in perenne interazione tra loro: non sono cioè soltanto delle 'aree' della mente, ma ne costituiscono anche i suoi fondamentali 'sistemi di funzionamento' perché, di fatto, ne attivano significative dinamiche ogni volta che entrano in contrasto tra di loro.

In particolare, la rimozione, secondo questa visione del primo Freud, è essenzialmente un processo che si svolge al confine fra il sistema Inconscio e quello preconscio: 'la sua essenza consiste semplicemente nell'espellere e nel tener lontano qualcosa dalla coscienza' (1915b, pag. 37). Essa, la cui finalità è quindi quella di tutelare l'equilibrio ed il benessere psicologico di ciascun individuo, va considerata, secondo Freud, una modalità universale dello psichismo umano, ed i suoi effetti sono facilmente evidenziabili anche nella comune vita quotidiana delle persone cosiddette 'normali' soprattutto in comportamenti quali i lapsus ed i cosiddetti atti mancati (Freud, 1904).

Il conflitto intrapsichico secondo Sigmund Freud negli anni della 'prima topica'

Secondo Laplanche e Pontalis, 'Nel quadro della prima teoria metapsicologica, il conflitto può essere fatto risalire schematicamente, dal punto di vista topico, all'opposizione fra i sistemi Inc e Prec, separati dalla censura; questa opposizione corrisponde anche alla dualità del principio di piacere e principio di realtà, in cui il secondo cerca di garantire la propria superiorità rispetto al primo. Si può dire che le due forze in conflitto sono allora per Freud la sessualità e un'istanza rimovente che include le aspirazioni etiche ed estetiche della personalità; la rimozione sarebbe motivata da caratteri specifici delle rappresentazioni sessuali che le renderebbero conciliabili con l' 'Io' e generatrici di dispiacere per quest'ultimo' (Laplanche \& Pontalis, 1967).

Il conflitto intrapsichico, in altri termini, è per Freud sin dall'inizio (e lo sarà sempre, anche se assumerà nel corso dei decenni delle connotazioni specifiche tra loro differenti) un conflitto tra pulsioni. Negli anni della prima topica, in particolare, si tratta del conflitto tra le pulsioni sessuali ed un gruppo di pulsioni ad esse opposte che risponde ad esigenze di sopravvivenza dell'individuo e di gratificazione di altre esigenze biologiche diverse dalla sessualità, e che Freud definirà, a fasi alterne, come Pulsioni di autoconservazione oppure anche come Pulsioni dell'Io.

Nella Figura 1 viene pertanto schematicamente rappresentata la visione del conflitto intrapsichico secondo Sigmund Freud durante gli anni della prima topica. 


\section{Il 'Complesso di Edipo'}

Questo costrutto teorico, che Freud ha tratto direttamente dalla propria pratica clinica e che sicuramente rappresenta una delle più note innovazioni freudiane, prende il nome dal mitologico re di Tebe, protagonista di una tragedia di Sofocle (1' 'Edipo re'), che ebbe l'inconsapevole e sciagurata sorte di uccidere il proprio padre biologico (Laio) e di congiungersi quindi sessualmente con la propria madre (Giocasta).

Nella vita psichica individuale esiste infatti un vissuto, secondo Freud universalmente diffuso, che si sviluppa nel periodo all'incirca compreso tra $i$ tre $\mathrm{e} i$ cinque anni di età e che consiste, essenzialmente, in un attaccamento libidico verso il genitore di sesso opposto, accompagnato da un atteggiamento ambivalente (e cioè di 'odio' ed 'amore' fusi insieme) nei confronti del genitore dello stesso sesso.

Il 'superamento del complesso di Edipo' è, secondo Freud, assolutamente fondamentale per la successiva evoluzione psicologica del soggetto, per il suo equilibrio o per l'eventuale instaurarsi, se esso non avviene in modo adeguato, di significative forme di psicopatologia (soprattutto di tipo nevrotico). Lo stesso Freud, del resto, aveva individuato nel complesso edipico 'il nucleo delle nevrosi' (Freud, 1915-17, 1932).

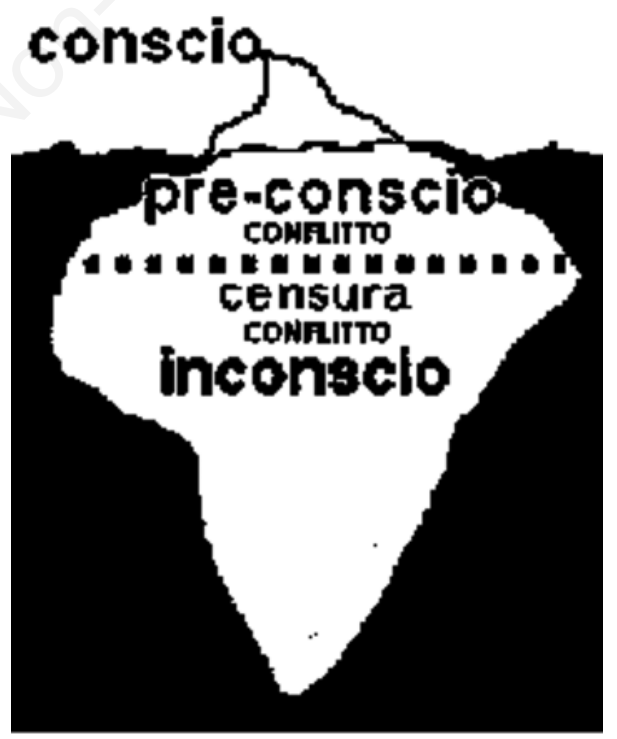

Figura 1. Il conflitto intrapsichico secondo Sigmund Freud negli anni della 'prima topica'. 
La psicoanalisi secondo Sigmund Freud

Nel suo scritto Due voci di enciclopedia: 'Psicoanalisi'e 'Teoria della Libido' (1922), Sigmund Freud fornisce in prima persona la propria visione della disciplina scientifica da lui fondata. Entrambe queste due definizioni furono pubblicate, nel 1923, rispettivamente alle pagg. 377-383 e alle pagg. 296-298 dell'Handwörterbuch der Sexualwissenschaft (Dizionario di sessuologia) stampato a Bonn, a cura di Max Marcuse, dall'Editore berlinese Walter de Gruyter.

Secondo lo stesso Sigmund Freud, quindi, 'psicoanalisi è il nome: i) di un procedimento per l'indagine di processi psichici cui altrimenti sarebbe impossibile accedere; ii) di un metodo terapeutico (basato su tale indagine) per il trattamento dei disturbi nevrotici; iii) di una serie di conoscenze psicologiche acquisite per questa via che gradualmente si assommano e convergono in una nuova disciplina scientifica' (Freud, 1922a).

\section{La terapia psicanalitica negli anni della 'prima topica'}

Negli anni della 'prima topica', per Freud il principale compito della terapia psicoanalitica è quello di 'Far riemergere alla coscienza il conflitto psichico rimosso' (cioè allontanato dalla coscienza perché spiacevole o doloroso).

Le principali tecniche introdotte da Freud sin dagli anni della 'prima topica' per aiutare il paziente a superare le resistenze che impediscono al rimosso di accedere alla coscienza sono in particolare le seguenti: i) ipnosi; ii) interpretazione dei sogni; iii) libere associazioni.

\section{La struttura della psiche ('seconda topica')}

Più o meno nello stesso periodo in cui coniava la definizione di Psicoanalisi sopra riportata, Freud operava una modificazione estremamente significativa del suo 'modello di funzionamento della mente' introdotto con la sua 'prima topica', proponendo con il libro L'Io e l'Es (1922b) un nuovo schema di descrizione dell'apparato mentale che, senza abbandonare la distinzione tra 'conscio', 'preconscio' e 'inconscio' che era alla base della sua concettualizzazione precedente, la integrava e modificava con la sistematizzazione in un unico 'insieme funzionale' costituito da tre 'sistemi' (definibili anche come 'istanze', dimensioni', 'entità', 'poli', ecc.)

Secondo il modello proposto da Freud con la sua 'seconda topica', pertanto, la psiche è un'unità complessa costituita da tre dimensioni (con funzioni diverse) in rapporto tra di loro: i) Es: polo pulsionale della personalità; Super- 
Io: coscienza morale; Io: parte organizzata e cosciente della personalità, che deve fare i conti con le esigenze dell'Es, del Super-Io e del mondo esterno.

Con l'introduzione della sua 'seconda topica', inoltre, Freud completa una prima revisione (peraltro già in corso da alcuni anni, e già evidenziatasi soprattutto nel suo volume del $1920 \mathrm{Al}$ di là del principio di piacere) della sua complessiva 'teoria delle pulsioni'.

\section{La rimozione nella 'seconda topica'}

Rimozione $=$ l'Io allontana dalla coscienza: i) gli eventi traumatici infantili (fonte di sofferenza e angoscia); ii) ogni pulsione che confligge: con il mondo esterno; o con le norme sociali interiorizzate (Super-Io).

Inoltre: i) il conflitto insito nella rimozione può produrre sintomi psiconevrotici; ii) i disturbi psichici presuppongono una rimozione.

Il conflitto intrapsichico nelle nevrosi e nelle psicosi dopo l'introduzione della 'seconda topica'

Secondo Laplanche e Pontalis (1967, pag. 93), 'Nel corso dell'opera freudiana, il problema del fondamento ultimo del conflitto ha ricevuto diverse soluzioni, Va notato innanzitutto che il conflitto può essere spiegato a due livelli relativamente distinti: al livello topico, come conflitto tra sistemi o istanze, e al livello economico-dinamico, come conflitto tra pulsioni'. Specificano inoltre, al riguardo, questi due stessi Autori: 'la seconda topica fornisce un modello più diversificato e più vicino a queste modalità concrete: conflitti tra istanze, conflitti interni a un'istanza, per esempio tra i poli di identificazione paterno e materno, che si possono ritrovare nel Super-io' (idem, pag.94).

Come si vede, quindi, il conflitto intrapsichico e il suo approccio da parte della psicoanalisi dopo l'introduzione della 'seconda topica' si complessifica in modo molto netto: Freud affronta questo tema in vari suoi scritti, proponendo dapprima (ad iniziare appunto dal suo volume L'Io e l'Es) una più semplice conflittualità tra ognuna di queste istanze e ciascuna delle altre due, oltre che tra contenuti interni ad ogni singola istanza, ma poi prendendo anche in esame la possibilità che ognuna delle tre istanze psichiche teorizzate dalla 'seconda topica' possa in un certo senso 'allearsi' con una delle altre due per avere il sopravvento rispetto alla terza.

Per riportare in un ambito di maggiore semplicità l'approccio teorico e metodologico psichico al problema del conflitto intrapsichico all'interno del modello psicoanalitico freudiano, Brenner (1976) proporrà la seguente conclusione: 
'Possiamo dire, molto in generale, che i conflitti sono di tre tipi. Nel primo, abbiamo un contrasto tra uno o più derivati istintuali e la parte più matura, organizzata e coerente della psiche: in questi casi il Super-io, se è già formato, sta dalla parte delle istanze psichiche più organizzate e coerenti. Nei conflitti del secondo tipo, la parte organizzata della personalità è in lotta con un bisogno inconscio di autopunizione. Infine, ci sono le situazioni in cui la tendenza autopunitiva si allea con un desiderio masochistico ed entrambi entrano in conflitto con la parte più matura e organizzata della psiche. Nel linguaggio della teoria strutturale, quindi, i conflitti sono tra Es ed Io (+ Super-io), tra Io e Super-io, tra Es + Super-io ed Io' (Brenner, 1976).

Poco dopo la pubblicazione di L'Io e l'Es, inoltre, nel suo saggio Nevrosi e Psicosi (1923), Freud arricchisce il suo contributo scientifico alla psicopatologia ed alla teoria del conflitto con quella che egli definisce come una 'semplice formula', ma che in realtà ha poi aperto approfondimenti enormemente vasti ed importanti all'interno del dibattito psicoanalitico delle epoche successive, riguardo alla principale differenza eziopatogenetica tra le nevrosi e le psicosi. Afferma infatti egli al riguardo: 'la nevrosi sarebbe l'effetto di un conflitto tra l'Io e il suo Es, mentre la psicosi rappresenterebbe l'analogo esito di un perturbamento simile nei rapporti tra Io e modo esterno' (Freud, 1923). Freud, nel medesimo saggio, completa poi questa sua affermazione con una specificazione ulteriore: 'la nevrosi di traslazione corrisponde al conflitto tra l'Io e l'Es, la nevrosi narcisistica a quello tra l'Io e il Super-io, la psicosi a quello tra l'Io e il mondo esterno' (Idem, p. 614).

La seguente Figura 2 schematizza, pertanto, la visione complessiva del

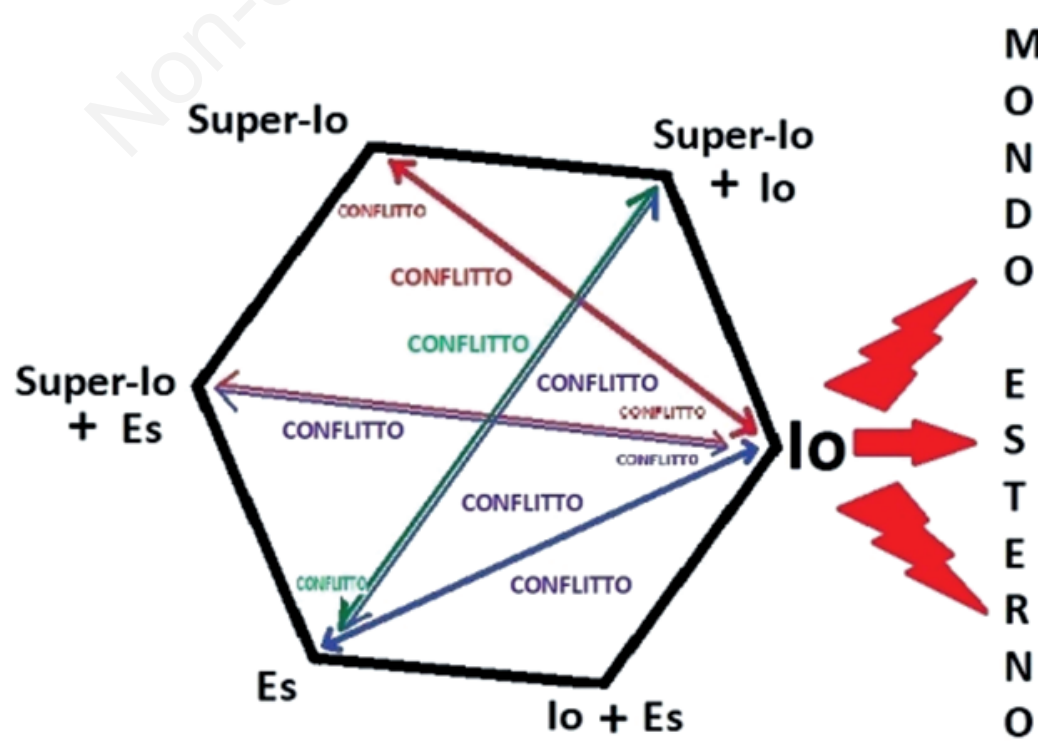

Figura 2. Il conflitto intrapsichico secondo Sigmund Freud negli anni della 'seconda topica'. 
conflitto intrapsichico che Sigmund Freud evidenziò negli anni della sua 'seconda topica'.

In questi stessi anni del suo passaggio dalla Prima alla seconda topica, inoltre, Freud progressivamente abbandonò la sua visione delle 'due specie di pulsioni' che aveva in precedenza considerato come suddivise nei gruppi delle 'pulsioni sessuali' e delle 'pulsioni autoconservative' (o 'pulsioni dell'Io'), conservando sostanzialmente le prime (che inizia anche a definire con il termine greco di Eros) ma sostituendo le seconde con le cosiddette 'pulsioni aggressive' (al cui interno egli colloca anche la cosiddetta 'pulsione di morte', che più tardi, a sua volta, definirà anche con il termine greco di Thanatos). Egli giustifica l'abbandono del suo precedente concetto di 'pulsioni autoconservative' con la considerazione secondo la quale 'entrambe le pulsioni agirebbero in modo conservativo, nel senso più rigoroso di questo termine, poiché mirerebbero al ripristino di uno stato turbato dall'apparire della vita. L'apparire della vita sarebbe dunque la causa della continuazione della vita e al tempo stesso dell'aspirazione alla morte; e la vita stessa sarebbe una lotta e un compromesso fra queste due tendenze' (Freud, 1922b).

Il concetto di 'conflitto intrapsichico' ed il suo trattamento psicoterapeutico nella concettualizzazione complessiva di Melanie Klein e della sua scuola

Melanie Klein (Vienna, 1882 - Londra, 1960) è stata una psicoanalista formatasi dapprima in Austria e quindi a Londra, dove è divenuta tra le personalità più decisive e influenti del movimento psicoanalitico mondiale di tutti i tempi. Ella è oggi diffusamente conosciuta in particolare per i suoi lavori pioneristici nel campo della psicoanalisi infantile e per suoi i contributi allo sviluppo della teoria delle relazioni oggettuali; la sua teoria complessiva è caratterizzata soprattutto dal valore estremamente concreto che ella attribuisce alle tre istanze psichiche che caratterizzano il modello della seconda topica freudiana (Io, Es e Super-io), dall'approfondimento dello studio dei meccanismi di difesa più primitivi ed arcaici già individuati da Freud (soprattutto la scissione, l'introiezione e la proiezione) e dall'individuazione di altri meccanismi di difesa inconsci la cui origine è per questa Autrice riconducibile alle prime fasi dello sviluppo psichico umano.

Sarebbe in particolare la 'fantasia inconscia' quella fondamentale attività che, all'interno del lungo processo fisiologico di crescita psicologica ed emozionale che caratterizza l'età evolutiva, crea e mantiene secondo la Klein una sorta di 'ponte' tra la mente e il corpo, i cui percorsi di sviluppo psichico e fisico risultano reciprocamente connessi e sufficientemente integrati ed armonizzati: nelle vicende individuali caratterizzate da espe- 
rienze di tipo psicopatologico tale collegamento risulterebbe invece disarmonico ed alterato, se non, in taluni casi, addirittura interrotto. L'estrema conseguenza di questa nuova teorizzazione kleiniana è che anche il 'conflitto tra l'Io e il mondo esterno' che era stato posto da Freud alla base della psicosi (Nevrosi e Psicosi, 1923) viene invece di fatto ridefinito dalla Klein come un conflitto primariamente interno al 'mondo oggettuale inconscio', e che solo secondariamente a ciò - nelle sue forme più intensamente caratterizzate dalla presenza attiva dell'aggressività e dell'istinto di morte - può assumere anche la caratteristica di conflitto con la realtà sottolineata da Freud.

E questo, a mio avviso, non è affatto un elemento secondario nell'evoluzione della Psicoanalisi come metodo psicoterapeutico, ma anzi ne costituisce probabilmente sul piano operativo la più importante novità di quei primi decenni della sua storia perché, differentemente da quanto esplicitato da Freud nella definizione che egli stesso fornì nel 1922 (e che di fatto mantenne sino alla sua scomparsa), riconduce invece anche le psicosi all'interno del gruppo di patologie affrontabili e trattabili con la tecnica psicoanalitica anche se, ovviamente, opportunamente modificata.

In un primo momento, la Klein utilizzò il metodo psicoanalitico nella terapia delle psicosi specificatamente nei bambini, integrando (e spesso anzi sostituendo) la tecnica delle libere associazioni verbali con la tecnica del gioco infantile. Successivamente, sia lei che i suoi allievi estesero il metodo psicoanalitico anche alla terapia delle psicosi degli adulti: e tutto ciò, a mio avviso, può con ogni probabilità essere visto anche come una delle principali conseguenze dell'aver riportato ogni forma di psicopatologia ai vari e diversificati conflitti attivi all'interno dell' apparato mentale, sia tra le tre istanze psichiche descritte da Freud con la sua 'seconda topica', sia tra parti scisse dell' Io, sia tra le varie forme di pulsioni di entrambe le specie ('di vita' e 'di morte') costantemente alimentate dal substrato biologico di ciascun essere umano sia, infine e soprattutto, dalle caratteristiche degli oggetti interni, delle loro rappresentazioni e caratterizzazioni affettive $\mathrm{e}$, last but not least, delle loro reciproche relazioni inconsce. Tutto ciò viene schematicamente rappresentato nella seguente Figura 3.

Grazie al contributo di Melanie Klein, pertanto, il fine principale delle psicoterapie psicoanalitiche si è spostato dalla 'cura dei sintomi' allo 'sviluppo della personalità', attraverso la reintegrazione delle scissioni nel Sé, la ricaratterizzazione affettiva degli oggetti interni ed il ritiro delle identificazioni proiettive delle parti infantili.

La psicoterapia quindi, nell'impostazione psicoanalitica kleiniana, appare orientata soprattutto verso la ricostruzione del 'mondo interiore' del paziente, affinché esso divenga il più possibile popolato da 'oggetti buoni' e risulti così di più facile ed armonica integrazione nel complessivo equilibrio psicologico e psicofisico di quest'ultimo. 
Il concetto di 'conflitto intrapsichico' ed il suo trattamento psicoterapeutico nella concettualizzazione complessiva di William Ronald Dodds Fairbairn

Al pari della Klein, di cui può essere sicuramente considerato un seguace e continuatore sia pure limitatamente alla prima parte della propria attività professionale e scientifica, William R. D. Fairbairn non smetterà mai di sottolineare la necessità, per lo psicoanalista concretamente dedito all'attività clinica, di relazionarsi con ogni proprio paziente considerandolo sempre come una 'persona' in carne ed ossa, portatrice di vissuti e di bisogni estremamente concreti con i quali il professionista non può non relazionarsi con altrettanta concretezza, con estremo rispetto e con la necessaria sensibilità.

Fairbairn accetta senza particolari riserve, evidentemente condividendone gli aspetti generali, il modello della 'prima topica' freudiana: nella sua impostazione teorica complessiva i concetti di 'Conscio', 'Preconscio' ed 'Inconscio' vengono infatti ampiamente utilizzati. Egli, tuttavia, delinea un'organizzazione strutturale e funzionale dell'Inconscio molto diversa da quella prospettata da Freud.

Secondo Fairbairn, l'Inconscio è sicuramente alla base della vita psichica di ogni persona, in quanto è un 'mondo interno' che contiene oggetti interiorizzati con cui le componenti strutturali più profonde del nostro Io hanno continuamente dei rapporti intensamente emotivi. In particolare, le parti dell'Io che costituiscono l'inconscio sono, oltre ad una parte significativa dell' Io Centrale' che permane quale residuo dell'Io originario, soprattutto i cosiddetti 'Io Libidico' ed 'Io Antilibidico' (per il significato di 'Io

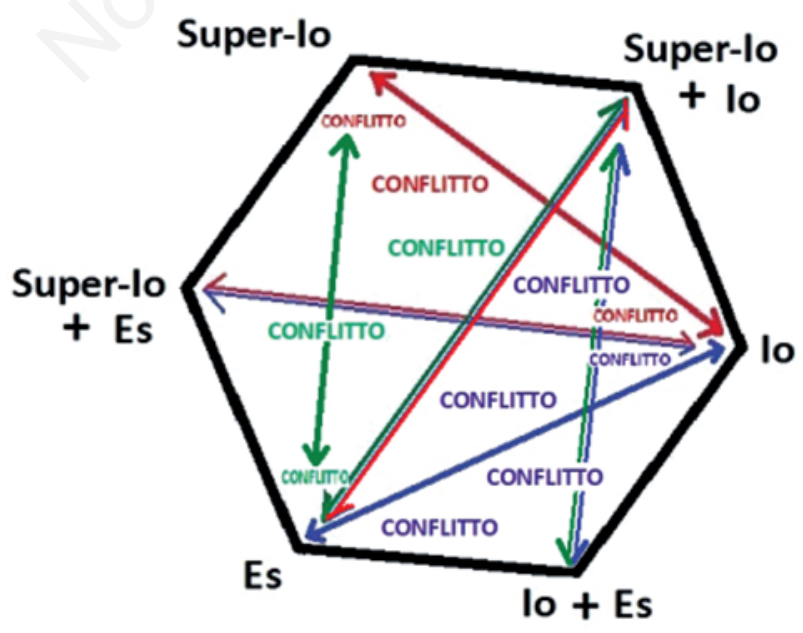

Figura 3. Rappresentazione schematica del conflitto intrapsichico nella teorizzazione di Melanie Klein. 
Libidico' e di 'Io Antilibidico', vedi oltre) che già durante la prima e la seconda infanzia hanno il compito di regolare i rapporti dell'Io con gli oggetti interiorizzati (genitori e altre figure significative). Di conseguenza l'inconscio presenta solo modelli immaturi di rapporto che, però, andranno a influenzare profondamente le relazioni oggettuali che ogni essere umano, programmato sin dalla nascita ad attivare, instaurerà col mondo esterno non solo nell'infanzia, ma anche per tutto il corso della propria esistenza.

Spingendo al limite estremo la visione kleiniana di un Inconscio originariamente concreto, fisico, praticamente 'materiale', Fairbairn giunge di fatto a teorizzare, a tale livello, l'equivalenza tra 'struttura' e 'oggetto'. È infatti proprio nell'inconscio che vengono a suo avviso ad essere interiorizzate e mantenute estremamente 'vive' le relazioni che il bambino instaura e consolida con i genitori durante i primi anni di vita.

Tali modelli, in una fase più matura, verranno esteriorizzati alle situazioni del mondo esterno sociale. L'individuo, secondo Fairbairn, si troverà così continuamente esposto alla difficile situazione di essere legato a relazioni immature con gli oggetti interiorizzati a livello inconscio mentre, d'altra parte, cerca di instaurare relazioni mature con gli oggetti a livello cosciente e razionale. Le 'relazioni oggettuali' sono, quindi, la più importante caratteristica (si potrebbe anzi dire le 'protagoniste assolute') dell'inconscio così come esso è concepito e descritto da Fairbairn.

\section{La rimozione secondo Fairbairn}

La rimozione, secondo Fairbairn, è in primo luogo un rifiuto dell'oggetto 'cattivo' da parte dell'Io originario: 'Ciò che viene primariamente rimosso non sono né le intollerabili pulsioni colpevoli, né i ricordi troppo spiacevoli, ma gli oggetti interiorizzati intollerabilmente cattivi' (Fairbairn, 1943).

Ciò provoca una scissione dell'Io originario e una conseguente rimozione delle parti dell' Io che sono direttamente collegate con gli oggetti rimossi, le quali daranno origine a due nuove strutture intrapsichiche che Fairbairn rispettivamente denomina come 'Io Libidico' e 'Io Antilibidico' (struttura psichica, quest'ultima, che nella prima versione della propria teoria Fairbairn aveva denominato con il significativo termine di 'sabotatore interno', in quanto tende a impedire al soggetto di consolidare i propri impulsi libidici in stabili relazioni positive con ciò che la Klein definirebbe con il termine di 'oggetti buoni'). Ciò che resta dell'Io originario dopo questa scissione viene denominato da Fairbairn come 'Io Centrale'.

Al contrario di quanto sosteneva Freud, infatti, per Fairbairn il rifiuto e la rimozione da parte dell'Io ancora non scisso non sono dirette solo verso gli oggetti 'cattivi', indipendentemente dal fatto che essi siano tali in quanto 'rifiutanti' oppure eccessivamente 'eccitanti', ma anche verso le parti 
dell'Io sussidiarie che sono collegate agli oggetti da rapporti di tipo libidico (e, quindi, oggetti 'buoni').

Secondo Fairbairn, possiamo quindi definire l'attacco dell'Io Centrale agli Io sussidiari con il termine di 'rimozione diretta': ma questa non è sufficiente per rimuovere l'oggetto eccitante, ed è per questo che in Fairbairn si ritrova anche il riferimento a una 'rimozione indiretta' da parte dell'Io Antilibidico nei confronti dell' 'Io Libidico.

La 'rimozione diretta', secondo Fairbairn, sarebbe quindi attivata dall'Io Centrale nei confronti sia dell' Io Libidico che dell'Io Antilibidico; quest'ultimo, inoltre, attiverebbe a sua volta nei confronti dell'Io Libidico una rimozione ulteriore, che Fairbairn definisce come 'rimozione indiretta', a supporto dell'Io Centrale e contro l'Io libidico. L'Io Antilibidico, nel modello di Fairbairn, collabora dunque con l'Io Centrale nell'attacco all'Io Libidico in uno schema dinamico nel quale chiaramente si evidenzia che, mentre la rimozione diretta avviene nei confronti sia dell'Io Libidico che dell'Io Antilibidico, la rimozione indiretta avviene invece soltanto nei confronti dell'Io Libidico.

Le componenti libidiche in eccesso sono dunque soggette secondo Fairbairn ad una misura di rimozione molto maggiore di quelle aggressive e, inoltre, è per lui evidente come la rimozione si attivi originariamente già nella fase schizoide piuttosto che in quella depressiva. Si deve pertanto inevitabilmente presumere una scissione precoce dell'Io originario, per giustificare il fatto che una parte di questa struttura psichica, ovviamente fornita di una carica di energia psichica dinamica, possa rimuovere un'altra parte di sé stessa dotata anch'essa di una carica di energia psichica dinamica.

\section{Le 'relazioni oggettuali' come base fondamentale dello sviluppo psichico individuale}

Le basi per una teoria oggettuale sono state gettate dalla Klein, la quale però rimane ancora saldamente ancorata alla teoria duale degli istinti come proposta da Freud. È invece a partire dagli anni 1940 che Fairbairn mette in atto un vero e proprio 'cambio di paradigma', riassumibile nella sua 'rivoluzionaria' affermazione secondo la quale 'lo scopo finale della libido è l'oggetto' (Fairbairn, 1941), o anche come dirà più tardi, 'Il vero fine libidico è l'instaurazione di relazioni soddisfacenti con gli oggetti; ed è quindi l'oggetto che costituisce la vera meta libidica' (Fairbairn, 1946). L'eziologia della psicopatologia deriva quindi sostanzialmente, secondo questo Autore, da disturbi delle relazioni oggettuali durante tutte le fasi di sviluppo, in quanto, in assenza di lesioni che compromettano la normale funzionalità dell'organismo, nell'essere umano i fattori ambientali hanno un'importanza primaria rispetto sia all'azione delle pulsioni sia ad altri ipotetici fattori innati o costituzionali. 
Il modello oggettuale/relazionale di Fairbairn della struttura della psiche umana e le sue implicazioni con il processo psicoterapeutico

Fairbairn abbandona completamente l'utilizzo dei concetti di Es, Io e Super-Io come erano stati originariamente utilizzati da Freud, e quindi anche l'uso di tali termini che ne fa la Klein: di fatto, pertanto, rifiuta completamente il modello ipotizzato dalla cosiddetta 'seconda topica' freudiana. Inoltre, egli sostiene che i primi oggetti a essere interiorizzati siano quelli che la Klein definirebbe come 'cattivi', e che ciò avvenga come forma di controllo della 'frustrazione libidica' generalmente associata all'esperienza dello svezzamento.

La relazione di ambivalenza con l'oggetto (la madre) che si presenta con l'avvento della fase orale tardiva viene a essere gestita dal bambino tramite tecniche complementari di scissione e di integrazione. In particolare, l'Io, che secondo questo Autore inizialmente è integro, scinde la figura della madre in oggetto 'buono' e oggetto 'cattivo', interiorizzando quest'ultimo nel tentativo di governarlo.

Per meglio controllare l'oggetto 'cattivo' dopo averlo introiettato, il bambino lo scinde secondo Fairbairn in 'oggetto eccitante' e in 'oggetto rifiutante', e utilizza per la rimozione di tali oggetti la propria aggressività attivata dalla frustrazione. Essa, però, solitamente in tale processo non si esaurisce del tutto: pertanto un'ulteriore dose di aggressività viene ad essere utilizzata da parte dell'Io centrale per scindersi in parte, specularmente, in un 'Io Libidico' e in un 'Io Antilibidico'.

La differenza tra l'Es di Freud e l'Io Libidico di Fairbairn consiste nel fatto che quest'ultimo non è un'originaria 'riserva di pulsioni', ma una vera e propria 'struttura psichica' derivata dal nucleo originario dell'Io centrale (e quindi più infantile e primitiva rispetto a ciò che quest'ultimo rappresenterà nell'organizzazione psichica complessiva dell'individuo adulto). La rimozione per Fairbairn si manifesta, come già in questa sede indicato, non solo verso gli oggetti (rimozione diretta primaria), ma anche direttamente verso questi due 'Io sussidiari', che restano legati agli oggetti da rapporti di tipo libidico (l'Io Libidico con l'oggetto 'eccitante'; l'Io Antilibidico con l'oggetto 'rifiutante').

Ovviamente, per Fairbairn, tale processo psichico di rimozione degli oggetti eccitanti e rifiutanti non è mai totalmente completato, ma deve essere continuamente riaffrontato. Quindi l'Io centrale opera, tramite una spinta di tipo aggressivo originata dalle frustrazioni subite, una rimozione diretta ('secondaria') rivolta sia all'Io libidico che all'Io antilibidico: quest'ultimo, utilizzando l'energia derivante dalla propria aggressività residua, mantiene attiva verso l'Io libidico un'ulteriore forma di allontanamento di quest'ultimo dalla coscienza (processo che viene definito da Fairbairn come rimozio- 
ne 'indiretta'), il che non solo dà origine ai fenomeni già noti come 'resistenza' all'interno del processo psicoterapeutico, ma contribuisce anche attivamente, nella vita psichica quotidiana, al mantenimento del meccanismo della rimozione verso l'Io libidico.

In sintesi, quindi, la 'situazione endopsichica fondamentale' proposta da Fairbairn vede un Io centrale, residuo dell'Io originario e più evoluto rispetto ad esso, che in parte è conscio, in parte maggiore è preconscio ed in parte ancora maggiore è inconscio, il quale interagisce continuamente con due Io sussidiari, ambedue completamente inconsci, denominati rispettivamente 'Io antilibidico' ed 'Io libidico'. Questi ultimi verrebbero originati dall'introiezione dell"'oggetto rifiutato' preambivalente e dalla successiva scissione da parte dell' Io di tale oggetto introiettato in 'oggetto rifiutante' ed 'oggetto eccitante', e mantenuti inconsci dall'azione della rimozione diretta ed indiretta.

Questa organizzazione dell' Io è quella che viene definita da Fairbairn $(1944,1946)$ come 'struttura endopsichica fondamentale' o 'struttura dinamica': essa si basa, ai fini della regolazione della libido e dell'aggressività, sull'antica tecnica del 'divide et impera' (Fairbairn, 1944, 1946). Egli, dopo averlo introdotto, apporterà a questo modello complessivo dell'organizzazione psichica alcune successive modifiche, e presenterà infine una dettagliata descrizione di questa sua visione finale della struttura della psiche umana nella Synopsis of an Object-Relations Theory of Personality, (1963), reperibile anche in lingua italiana alle pagg. 169-170 del suo volume Il piacere e l'oggetto: scritti 1952-1963 con il titolo Sinossi della teoria delle relazioni oggettuali applicata alla personalità.

Fairbairn specifica al riguardo, tra l'altro, che a suo avviso:

'1) Un Io è presente fin dalla nascita.

2) La libido è una funzione dell'Io.

[...]

9) Due aspetti dell'oggetto interiorizzato, ossia quello eccitante e quello frustrante, vengono scissi dal nucleo principale dell'oggetto e rimossi dall'Io.

10) Si costruiscono così due oggetti interni rimossi, l'oggetto eccitante (o libidico) e l'oggetto rifiutante (o antilibidico).

[...]

12) Poiché l'oggetto eccitante (libidico) e quello rifiutante (o antilibidico).sono entrambi investiti dall'Io originario, essi portano con sé nella rimozione anche le parti dell'Io da cui sono investiti, mentre il nucleo centrale dell'Io (Io centrale) non viene rimosso ed è l'agente della rimozione.

13) La situazione interna che ne consegue è costituita dall'Io originario scisso in tre strutture egoiche: un Io centrale (cosciente) connesso all'oggetto ideale (ideale dell'Io), un Io libidico rimosso connesso all'oggetto eccitante (o libidico) e un Io antilibidico rimosso connesso all'oggetto rifiutante (o antilibidico) ' (pp. 169-170, Fairbairn, 1963).

Questa strutturazione della psiche è rappresentata graficamente nella seguente Figura 4. Le frecce nere al suo interno rappresentano la dinamica 
della rimozione come descritta da Fairbairn nei suoi ultimi lavori.

Proseguendo nella descrizione particolareggiata del proprio modello della psiche, fondato sulle relazioni interiorizzate che ne costituiscono le fondamentali strutture, nella medesima Sinossi Fairbairn fornisce anche chiare indicazioni sulla conseguente dinamica dei conflitti intrapsichici umani:

'15) L'Io antilibidico, in virtù del suo legame con l'oggetto rifiutante (antilibidico), adotta un atteggiamento assolutamente ostile verso l'Io libidico, e in tal modo ha l'effetto di rinforzare la rimozione dell'Io libidico da parte dell'Io centrale' (Idem, p. 170).

Questa dinamica è evidenziata in rosso nella seguente Figura 5 .

Tutto ciò, secondo Fairbairn, non si limita ad una revisione degli aspetti puramente teorici della Psicoanalisi freudiana e kleiniana, ma comporta anche significative revisioni delle concrete modalità di intervento psicoterapeutico. Egli così descrive il proprio pensiero al riguardo:

'Alla luce di queste considerazioni, sembra che per quanto lo psicoanalista terapeuticamente possa assegnare a sé stesso un ruolo neutrale, non possa sottrarsi alla necessità di intervenire attivamente se vuole essere terapeuticamente efficace, e si deve riconoscere che ogni interpretazione è realmente un intervento. Perciò, in un certo senso, il trattamento psicoanalitico si risolve nel tentativo da parte del paziente di confinare la sua relazione con l'analista nel sistema chiuso della realtà interna mediante il transfert, e nella determinazione da parte dell'analista di aprire una breccia in questo sistema chiuso e di offrire condizioni nelle quali, nel contesto di una relazione terapeutica, il paziente

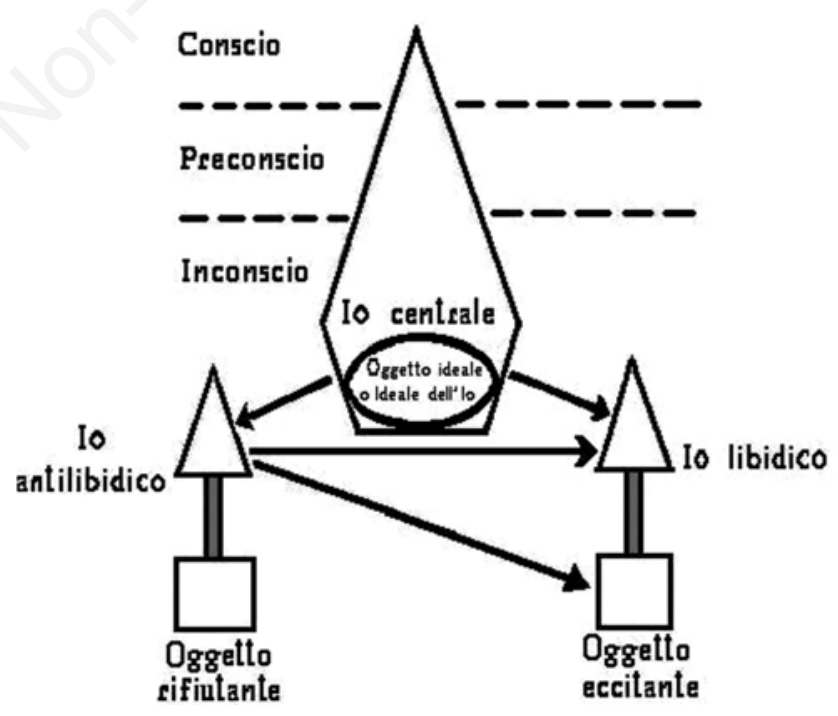

Figura 4. Il definitivo modello di Fairbairn sulla struttura della psiche umana (1963, in 1992). 
possa essere indotto ad accettare il sistema aperto della realtà esterna. La possibilità che l'analista raggiunga tale obiettivo dipende in misura non irrilevante da quanto rigidamente, in ogni singolo caso, la realtà esterna si sia strutturata come sistema chiuso. La valutazione di questo aspetto deve essere considerata il criterio reale per stabilire l'idoneità di un caso al trattamento psicoanalitico, Sembra comunque che, se le considerazioni che precedono sono fondate, la relazione reale tra paziente e analista costituisca il fattore decisivo della cura psicoanalitica, come di ogni altra forma di psicoterapia, anche se nel caso della terapia psicoanalitica agisce indubbiamente in maniera peculiare' (pp. 167168, Fairbairn, 1958).

Il progressivo abbandono del modello psicoanalitico pulsionale a favore del nuovo modello relazionale

Attraverso i contributi sia teorici che metodologici non solo di Fairbairn, ma anche di moltissimi altri Autori che hanno definitivamente abbandonato il tradizionale modello pulsionale freudiano e kleiniano a favore del nuovo modello relazionale come base fondamentale sia per comprendere il sistema psichico complessivo degli esseri umani che per utilizzarlo come fondamento del loro approccio psicoterapeutico verso le persone, la Psicoanalisi ha completato un percorso di revisione e di trasformazione della propria identità originaria che in realtà era cominciato molti anni prima, e non soltanto in Europa, e che poi si è diffuso con caratteristiche anche molto specifiche e differenziate ormai in quasi tutte le aree geografiche del nostro Pianeta.

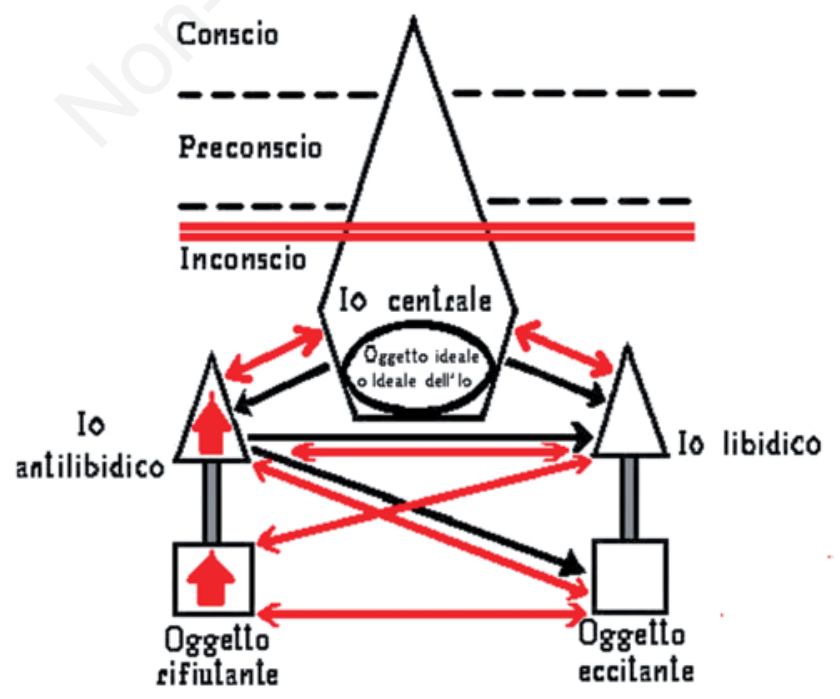

Figura 5. Il conflitto intrapsichico nel modello definitivo di Fairbairn sulla struttura della psiche umana. 
Spiega, al riguardo, l'illustre psicoanalista italiano Marco Bacciagaluppi:

\begin{abstract}
'Il modello relazionale ebbe origine con Ferenczi. [...] L'influenza di Ferenczi si esercitò su entrambe le sponde dell'Atlantico, specialmente attraverso due donne analizzate da lui, Melanie Klein in Gran Bretagna e Clara Thompson negli Stati Uniti. [...] Melania Klein ... abbandonò il paradigma relazionale di Ferenczi e adottò un modello pulsionale ancora più avanzato di quello di Freud. [...] I membri della Middle School britannica, invece, si trovano chiaramente nella tradizione di Ferenczi per quanto riguarda il rapporto amorevole col paziente. Fairbairn (1940) parla di un 'contatto emotivo genuino' in terapia. Winnicott (1958) di un 'rapportarsi di base dell'Io'. [...] Un altro legame esplicito con Ferenczi in Gran Bretagna è rappresentato da Ian Suttie. [...] Negli Stati Uniti, Ferenczi contribuì alla creazione della scuola interpersonaleculturale, grazie a Clara Thompson, analizzata da lui, e a Fromm, suo grande ammiratore. [...] Senza le costrizioni dell'ortodossia freudiana, la scuola interpersonale-culturale americana, fondata più tardi da Fromm, Sullivan e la Thompson, fu libera di sviluppare i temi ferencziani. [...] Tutti questi Autori si possono definire relazionali in senso lato. [...] Altri contributi in questo campo sono Mitchell (1988 e 1993), Skolnick e Warshaw (1992), Aron e Sommer Anderson (1998), Bromberg (1998/2001), Donnel Stern (1997, 2010) e Hirsch (2008)' (Bacciagaluppi, 2012).
\end{abstract}

E questo elenco di Autori afferenti al cosiddetto modello relazionale in senso ampio, sicuramente, potrebbe continuare ancora molto molto a lungo, ad esempio citando altri nomi di chiara fama internazionale quali ad esempio quello di Jay R. Greenberg (autore insieme a Mitchell dell'ormai 'classico' volume Le relazioni oggettuali nella teoria psicoanalitica, pubblicato in Italia nel 1986).

Conclude infine, al riguardo, Bacciagaluppi: 'è giustificato applicare il termine 'Psicoanalisi' al modello relazionale. Esso ha in comune con la Psicoanalisi classica i tre concetti fondamentali che Freud stesso riteneva necessari per definire una terapia come psicoanalitica: 1) l'inconscio 2) la resistenza 3) il transfert (Freud, 1914a)' (Idem, p. 165).

Della problematica del conflitto in ambito psicoanalitico, a partire però da punti di vista significativamente differenti rispetto a quelli che caratterizzano invece il filone 'relazionale', nel corso del dibattito degli anni 1960, 1970 e 1980 si sono diffusamente occupati anche vari altri Autori molto noti anche in Italia (ad esempio Wilfred Bion, Merton Gill, Robert Holt, George Klein, Joseph e Anne-Marie Sandler, ecc.) che pertanto, in questa sede, devono essere quantomeno citati.

Un approfondimento specifico dei loro apporti su questo tema va sicuramente ben oltre gli scopi del presente lavoro: mi sembra però utile quantomeno ricordarli perché, sebbene a partire da diverse angolazioni, molti dei loro contributi giungono comunque ad esprimere, in modi almeno in parte a quelli provenienti dagli Autori di ambito più specificatamente relazionale, interessanti punti di convergenza tra la teoria del conflitto e l'intersoggettività 
da un lato (ad esempio con l'approfondimento di concetti come quelli di controtransfert, di setting e di campo) e i successivi contributi delle Neuroscienze dall'altro. Su alcuni di questi argomenti cercherò pertanto di ritornare nella parte conclusiva del presente lavoro.

Di fatto comunque, in questi più recenti decenni, il definitivo abbandono del modello psicoanalitico pulsionale da parte di un numero progressivamente sempre più consistente di importanti psicoanalisti sia europei sia americani, come rileva ad esempio Massimo Fontana (2016), ha avuto importanti ripercussioni non solo nella complessiva concezione del funzionamento della mente umana e nella netta valorizzazione della relazione reale tra paziente e analista al fine dell'esito della psicoterapia psicoanalitica, ma anche nel ruolo e nel significato attribuito al conflitto intrapsichico nell'eziologia dei disturbi e delle patologie mentali, il cui 'focus' si è anch'esso progressivamente spostato dalla dimensione intrapsichica a quella relazionale.

Nota infatti innanzitutto al riguardo questo Autore:

\begin{abstract}
'Riguardo all'eziologia, la tradizione freudiana (e della Psicologia dell'Io) porta con sé una concezione della psicopatologia come risultato delle lotte interne (dei conflitti intrapsichici) tra pulsioni radicate nel piano biologico e difese dell'Io. Mentre le teorie delle Relazioni Oggettuali (Fairbairn, Winnicott) $e$, per altri versi, la Psicologia del Sé (Kohut) e la tradizione interpersonale (Sullivan), vedono nelle carenti risposte ai bisogni infantili da parte delle figure di accudimento, o nella disfunzionalità delle relazioni reali con gli altri, la causa dei disturbi dello sviluppo psicologico' (Fontana, 2016).
\end{abstract}

Ma mentre in Europa, almeno nei primi due o tre decenni successivi ai contributi di Fairbairn e soprattutto per opera degli Autori del cosiddetto Gruppo degli Indipendenti inglesi (gruppo che viene spesso anche definito come Middle Group o Middle School, e del quale alcuni tra i suoi i più noti esponenti sono ad esempio Winnicott, Guntrip, Kahn e Bowlby) la causa fondamentale dei disturbi psicologici viene decisamente indicata nelle carenti risposte ai bisogni infantili da parte delle figure di accudimento e nelle negative conseguenze che ciò comporta sulle capacità di relazione con gli altri e con la propria vita, negli Stati Uniti il focus sul nucleo delle problematiche psichiche si sposta gradualmente dalla dimensione diadica - o comunque circoscritta - del rapporto tra il bambino e la madre o tra il bambino e il suo nucleo famigliare ristretto a quello delle relazioni complessive che il singolo individuo instaura con tutte le altre figure significative lungo tutto il corso della sua esistenza.

Uno degli Autori statunitensi a cui maggiormente si deve questo cambio di prospettiva è sicuramente Stephen Mitchell (1988), che viene indicato da Massimo Fontana come colui che per primo, proprio sulla base del radicale cambio di paradigma avvenuto all'interno della Psicoanalisi nei decenni precedenti col passaggio dal modello pulsionale al modello relazionale, ha 
evidenziato la necessità, nell'ambito della comprensione delle dinamiche eziologiche delle problematiche psichiche, della sostituzione del concetto di conflitto intrapsichico con quello di conflitto relazionale.

È infatti proprio il concetto di conflitto relazionale introdotto da Mitchell, secondo Fontana, che consente il superamento della rigida e sterile contrapposizione tra conflitto intrapsichico (su cui si era focalizzata la Psicoanalisi pulsionale freudiana e kleiniana, ma anche quella già oggettuale su cui era costruito il modello di Fairbairn) e deficit relazionale che aveva invece costituito il nucleo delle teorie eziopatogenetiche degli Autori del Middle Group.

Ma anche un'altra e non meno significativa innovazione, secondo Fontana, ha contribuito a rendere ulteriormente significativa la Psicoanalisi relazionale americana nella sua efficacia esplicativa ed applicativa durante gli ultimi decenni del secolo scorso. Si tratta, in particolare, della parziale sostituzione del meccanismo di difesa della rimozione con quello della dissociazione per il funzionamento non solo patologico, ma anche normale, della mente umana.

'La riscoperta del concetto di dissociazione, collegata al risveglio dell'interesse nei confronti del trauma reale a partire dalla metà degli anni 1980, ha comportato quindi un certo scompiglio in psicoanalisi... [...] Tale scompiglio si può ridurre nei suoi fattori essenziali affermando che in psicoanalisi, attualmente, si confrontano due diversi modi di intendere la dissociazione: l'uno, risolve il problema riconducendola all'impostazione freudiana, e intendendola come uno dei tanti meccanismi di difesa e, nello specifico, come un modo con il quale l'individuo si protegge attivamente da memorie traumatiche vissute come intollerabili; l'altro, sempre più diffuso, segue proprio la prospettiva di Janet, considerando la dissociazione come la conseguenza di traumi psicologici che danneggiano le facoltà integrative della coscienza. Anche se non espresso dalla voce di uno psicoanalista, si può ricorrere ad un eloquente paragone di Liotti (1999) per rappresentare quest'ultima posizione: 'pensare alla dissociazione come a una difesa sarebbe analogo al considerare le fratture ossee come reazioni difensive a traumi fisici' (Idem, p. 6).

Pertanto, nella prospettiva della Psicoanalisi relazionale americana e grazie soprattutto alle specifiche innovazioni introdotte da Bromberg (1998/2001), 'la mente è intrinsecamente (e normalmente) strutturata sulla dissociazione, in quanto costituita fin dalla sua origine dalle molteplici configurazioni Sé/Altro che derivano dalle diverse relazioni interpersonali significative. La mente, dunque, sarebbe intrinsecamente dissociata perché fa inevitabilmente parte dello sviluppo di ogni individuo il costituirsi di molteplici Sé, ciascuno adeguato al proprio specifico campo relazionale e bisognoso di potersi esprimere pienamente per realizzare il proprio potenziale, consolidarsi e permettere il pieno e autentico coinvolgimento della persona in ogni relazione' (Idem, p. 6). 
Peraltro, vorrei sottolinearlo in modo particolare, questo recupero - operato come si è detto principalmente da Bromberg - della dissociazione come meccanismo difensivo fondamentale di risposta al trauma psichico almeno quanto la rimozione, si inserisce in modo assolutamente armonico sia nella precedente teorizzazione complessiva della Psicoanalisi 'tradizionale' freudiana sia nella specifica e più recente evoluzione teorica successiva che è invece caratteristica, in modo marcatamente originale, della psicoanalisi della relazione italiana.

Afferma infatti testualmente al riguardo Marco Bacciagaluppi, uno psicoanalista milanese molto vicino a Bowlby e a Fromm, in una sua intervista (rilasciata nel 1998 a Michele Minolli, tra i principali fondatori della Società Italiana di Psicoanalisi della Relazione e pubblicata sulla rivista Ricerca Psicoanalitica di cui la medesima Associazione è proprietaria e curatrice): 'In Attachment (Bowlby, 1969, pp. 10-11 dell'originale) Bowlby passa in rassegna la concezione che Freud aveva del trauma. Di Freud accetta sostanzialmente il concetto che un avvenimento è traumatico quando l'apparato mentale è sottoposto a quantità eccessive di stimolazione. Bowlby conclude che la separazione e la perdita 'sono semplicemente un esempio particolare del tipo di evento che Freud considerava traumatico' (op. cit., p. 11). Userò quindi la separazione traumatica come paradigma della situazione traumatica.

Delle tre fasi della risposta di un bambino alla separazione traumatica, la protesta è un'espressione dell'ansia di separazione, la disperazione è una manifestazione del lutto e il distacco è la conseguenza di una difesa da queste emozioni (Bowlby, 1973, p. 27 dell'originale). La difesa è costituita dalla negazione, dalla rimozione e, forse più spesso, dalla dissociazione. Bowlby (op. cit., p. 29) nota che questa stessa sequenza viene descritta da Freud alla fine di Inibizione, sintomo e angoscia (Freud, 1926).

Alla luce di quanto sopra, mi sembra di poter rispondere che è traumatico l'evento, mentre le difese tendono ad attenuarne le conseguenze, pur avendo, a loro volta, conseguenze negative sullo sviluppo della personalità' (Minolli, 1998).

È proprio nella sistematizzazione complessiva della psicoanalisi della relazione italiana che, almeno a mio avviso, questi contributi di varia provenienza storica sul conflitto e sulle difese che caratterizzano la natura e la Personalità umana si riunificano in un modello coerente sia sul piano teorico che su quello della sua validità in ambito applicativo, terapeutico.

In Italia, in particolare, il modello psicoanalitico relazionale inteso in senso complessivo ha iniziato a svilupparsi tra la fine degli anni Settanta e i primi anni Ottanta del secolo scorso, dando origine ad alcune Società e Scuole di Psicoanalisi ormai presenti in varie Regioni del nostro territorio nazionale e che, attraverso la produzione di contributi originali, hanno soprattutto cercato di costruire ognuna, in questi ultimi decenni, una propria specifica identità. Tra di esse, in particolare, mi soffermerò quindi ora su 
quanto ho reperito sul tema dell'interpretazione del conflitto e della sua relazione con le modalità tecniche e metodologiche adottate per affrontarlo in ambito psicoterapeutico attraverso il modello della cosiddetta psicoanalisi della relazione.

Le principali peculiarità di questo specifico approccio, rispetto ad altre impostazioni presenti all'interno del modello relazionale in senso ampio sia italiane che internazionali, consistono a mio avviso in due ulteriori elementi innovativi da essa adottati, vale a dire l'utilizzo in ambito psicoanalitico della teorizzazione anche più recente riguardante i cosiddetti sistemi dinamici complessi e la particolare attenzione alla relazione attuale tra psicoanalista e paziente quale 'chiave d'accesso privilegiata' sia verso la comprensione dei conflitti relazionali di quest'ultimo sia per la loro conseguente presa in carico ai fini psicodiagnostici e psicoterapeutici.

Il concetto di conflitto relazionale secondo l'odierna psicoanalisi della relazione e le sue conseguenze sulle modalità di trattamento psicoterapeutico

Tra i principali presupposti teorici dell'attuale Psicoanalisi della Relazione dobbiamo tenere presenti almeno i quattro seguenti: i) il superamento sia del concetto di conflitto intrapsichico sia di quello di deficit relazionale, a favore del concetto di conflitto relazionale; ii) la costante compresenza, nel processo di strutturazione e di funzionamento dinamico della mente umana, di almeno due meccanismi difensivi fondamentali, sia pur agenti in misura tra di loro differente e spesso associati con altri meccanismi ancora: la rimozione e la dissociazione; iii) la visione della Personalità umana come parte inscindibile di un complesso di sistemi dinamici complessi, con i quali è in costante relazione reciprocamente attiva; iv) la concezione della relazione psicoanalitica come espressione dei conflitti relazionali presenti all'interno del sistema complesso 'paziente-terapeuta' (o 'pazienti-terapeuta' nel caso di trattamento di coppia o di gruppo), e quindi della sua imprescindibile caratteristica di oggetto privilegiato dell'analisi.

Sulla base di questi quattro presupposti teorici, il modello esplicativo del funzionamento normale o disfunzionale del singolo individuo si fonda almeno sui cinque costrutti specifici seguenti: i) l' 'Io-soggetto'; ii) 1' 'autoeco-organizzazione'; iii) la 'coscienza della coscienza'; iv) la 'creatività'; v) la 'presenza a sé stessi'.

Tutti questi costrutti saranno ora ad uno ad uno dettagliati in modo più specifico secondo le definizioni proprie della psicoanalisi della relazione contemporanea.

Per quanto riguarda il primo di questi quattro costrutti, Michele Minolli (2015) specifica innanzitutto che: 
- L'Io-soggetto è uno - Affermare che l'Io-soggetto è uno coniuga alla radice la diversità con l'unità, le parti con il tutto, così da rispettare l'Io-soggetto in quanto tale e evitare l'assolutizzazione di una parte o una visione olistica dell'insieme; - L'Io-soggetto ha più parti in interazione tra di loro - Le varie componenti o le diverse funzioni devono essere colte nella loro interazione. Un modello che colga soltanto l'aspetto dell'unitarietà e non aiuti a capire il funzionamento ricorsivo del soggetto tra le sue parti e il tutto non è adeguato;

- L'Io-soggetto é in rapporto con l'esterno - Non avrebbe molto senso occuparsi di un Io-soggetto estraniato dalla realtà interattiva nella quale è necessariamente inserito. E questo, in particolare, rispetto alle influenze e alle modalità di incidenza retroattive nelle interazioni con l'oggetto esterno' (Minolli, 2015).

Riguardo al concetto di 'auto-eco-organizzazione', afferma invece in particolare Minolli: 'Due o più sistemi in interazione tra loro danno luogo a cambiamenti sempre determinati dall'unità che accoglie le perturbazioni. Sia il sistema sia l'ambiente risultano fonte di perturbazione reciproca ed è solo dal punto di vista di un osservatore esterno se il cambiamento del sistema viene pensato come determinato dall'ambiente o dall 'interno' (Minolli, 2009). Prosegue, al riguardo, il medesimo Autore: 'Qualsiasi considerazione che porti ad accentuare l'una o l'altra di queste incidenze è chiaramente dipendente dal punto di vista adottato. Se andiamo al di là, se cioè cerchiamo di considerare l'Io-soggetto per come si presenta a sé stesso e agli altri, egli è sempre il risultato di auto ed eco-organizzazione' (Idem, p. 53).

Precisa inoltre, al riguardo, Massimo Fontana:

\footnotetext{
'Questo dell'auto-organizzazione - o 'auto-eco-organizzazione', come preferiscono esprimersi Morin (1985) e Minolli (2009) per sottolineare il superamento della logica dualistica - è un principio cruciale di tale modo di inquadrare teoricamente lo sviluppo in una prospettiva relazionale: proprio grazie ad esso è possibile non scivolare in un interazionismo di superficie che, invece di cogliere le transazioni che avvengono nel campo relazionale, si limiti a ripartire fattori di sviluppo fra interno ed esterno, come se fossero realtà concepibili indipendentemente l'una dall'altra' (Fontana, 2016).
}

Come il principio della 'auto-eco-organizzazione' appare, pertanto, assolutamente fondamentale nel determinare la concezione dell' Io-soggetto come sempre inestricabilmente connesso con l'ambiente in cui nasce e vive, così i concetti di 'Coscienza della coscienza' e di 'Creatività', secondo Minolli, appaiono altrettanto legati all'originale principio della 'Presenza a se stessi', che questo Autore considera come il punto di riferimento fondamentale per una reale possibilità, peraltro per nulla scontata, di 'prendere in mano la propria vita'.

'Useremo 'coscienza' per indicare la capacità dell'essere vivente di cogliere l'esterno e l'interno e quindi la capacità di essere in rapporto, consciamente e inconsciamente, con sé stessi e con il mondo. Un cogliere e un essere in rapporto che implica, come dice Morin (1981, p. 268), un'azione 
cognitiva, una distinzione sé/non sé e, in qualche modo, un'auto-trascendentalizzazione del sé rispetto al non-sé. In questa luce 'coscienza' è una capacità di cui è dotato qualsiasi essere vivente: dalla cellula all'essere umano. Tralasciando il come funziona e quindi la coscienza cognitiva, ma anche il risultato e quindi la coscienza fenomenica, ci attestiamo esclusivamente sulla 'coscienza' come capacità di ogni vivente di operare una separazione tra sé e non sé.

Useremo 'Coscienza della coscienza' come la capacità specifica dell' Homo sapiens di occuparsi e pronunciarsi sulla 'coscienza'. Solo l'essere umano ha la prerogativa di questo salto qualitativo che lo porta a vedere la 'coscienza' domandandosi il perché e il che cosa farne' (Minolli, 2015).

Però, infine:

\begin{abstract}
'Per arrivare alla Presenza a sé stessi la 'coscienza della coscienza' affronta molti e inevitabili ostacoli: dal rifiuto alla reazione, dalla negazione all'idealizzazione, dalla rassegnazione alla rinuncia, dalla delega alla società al rifugiarsi nella patologia. Non è necessario spodestare alcuna Carta costituzionale per criticare e ritenere fuori legge queste circonvoluzioni che l'Io-soggetto mette in opera per raggiungere la qualità della Presenza a sé stesso. Una delle molle, forse la più incisiva, che spinge verso la Presenza è la sofferenza cioè il malessere di non potersi dire di essere sé stessi per quello che si è e di potere quindi prendere in mano la propria vita' (Idem, p, 233).
\end{abstract}

Sulla base di questa descrizione del processo verso l'acquisizione della 'Presenza a se stessi' come descritto da Minolli nel suo lavoro del 2015, nonché dei suoi quattro 'presupposti teorici' in precedenza declinati ed illustrati, l'applicazione al momento psicoterapeutico del modello della psicoanalisi della relazione si svincola - a mio avviso pressoché definitivamente non solo dal concetto di conflitto intrapsichico, ma anche dal concetto di modello ideale a cui tutte le persone debbano necessariamente tendere quando iniziano una psicoterapia secondo l'approccio dell'odierna psicoanalisi della relazione.

Conclude infatti, al riguardo, lo stesso Minolli (2009): 'Ogni sistema, quindi anche ogni sistema umano, segue la sua strada e trova le sue soluzioni. Qualunque sia il suo stato è certamente funzionale alla sua coerenza.

Non esiste un modello ideale di come dovrebbe essere. Non esiste un tempo del cambiamento. Non esiste un modo auspicabile di essere.

L'analisi può solo perseguire una 'Presenza a sé stesso' del sistema. Una Presenza a sé stessi che permetta di arrivare a stare 'bene' nelle proprie soluzioni storiche e quindi attuali o di proporsi cambiamenti verso direzioni stabilite dal sistema stesso.

La fisica, la biologia e le scienze dell'evoluzione ci sono di aiuto nel pensare un cambiamento funzione del sistema. È al servizio del sistema che noi operiamo. È come 'facilitatori' dell'Io-soggetto che siamo importanti' (Minolli, 2009). 


\section{Conclusioni}

La teorizzazione complessiva della scuola della psicoanalisi della relazione, come qui sommariamente riportata soprattutto attraverso la sintesi del contributo di Michele Minolli, sembrerebbe quindi sostanzialmente confermare quanto già affermato esplicitamente anche da Massimo Fontana, e cioè che anche per la psicoanalisi della relazione, come già era stato sottolineato da Mitchell nel suo volume del 1988, risulta confermata la necessità del completo superamento del concetto di conflitto intrapsichico (come pure di quello di deficit relazionale che aveva invece costituito il nucleo delle teorie eziopatogenetiche degli Autori del Gruppo degli Indipendenti Inglesi come ad esempio Fairbairn, Winnicott e Bowlby) a favore del concetto di conflitto relazionale.

Questo concetto, specifica al riguardo Massimo Fontana, 'considera l'organizzazione psicologica come necessariamente legata al campo relazionale, secondo un modello transazionale in cui le modalità di dare significato all'esperienza, e i significati stessi, vengono co-costruiti nell'interazione con l'altro. In questa ottica, non esiste un interno ed un esterno indipendenti l'uno dall'altro, ma un campo d'interazione nel contesto del quale prendono forma desideri e paure, ed ogni altro aspetto della vita psicologica; per Mitchell la regolazione del Sé procede insieme con la regolazione del campo' (Fontana, 2016).

Precisa inoltre, al riguardo, Salvatore Zito: 'In sostanza, ciò che la rivoluzione relazionale ha avuto come suo principale obiettivo è stato proprio quello di incidere sull'ottica mediante la quale la psicoanalisi indaga il soggetto psichico: non più un individuo isolato, separato, i cui desideri sono in intrinseco contrasto con la realtà esterna, ma un campo intersoggettivo all'interno del quale il soggetto nasce e psicologicamente si svilup$p a^{\prime}$ (Zito, 2017).

Il concetto di 'campo', in psicologia, vanta sicuramente una lunga tradizione, sin dai primi fondamentali contributi che espressero intorno ad esso Kurt Lewin e gli Autori aderenti alla teoria della Psicologia della Forma nella prima metà del secolo scorso. Esso era peraltro già stato utilizzato $\mathrm{e}$ considerato con straordinaria attenzione da parte di tutti e tre i modelli fondamentali della Fisica che si erano affermati sin dall'inizio non solo del XX secolo ma anche da quelli precedenti, vale a dire dal modello del 'campo gravitazionale' introdotto da Newton e poi radicalmente modificato da Einstein (1687-1916), da quello del 'campo elettromagnetico' di Faraday e Maxwell (1812-1864) ed infine da quello della cosiddetta 'teoria quantistica dei campi' introdotta a partire dal 1926 da Schrödinger e da Dirac e poi sviluppata da molti altri Autori tra i quali basterà citare in questa sede Heisenberg, Pauli e Oppenheimer (1926-1950 circa). In tempi più recenti, esso è stato più volte utilizzato - sia direttamente sia indirettamente - anche 
da molti Autori di ambito psicoanalitico: ne ritroviamo ad esempio un riferimento molto evidente, sia pure indiretto, nel concetto di simbiosi che è alla base della teoria della 'separazione-individuazione' formulata a partire dagli anni 1950 da Margaret S. Mahler. Successivamente esso è stato ripreso in modo implicito anche da Winnicott e da Bowlby (all'interno, rispettivamente, dei loro specifici concetti di 'diade madre-bambino' e di 'attaccamento'), ma poi è stato utilizzato in modo sempre più esplicito, soprattutto dagli autori di derivazione kleiniana e bioniana, con particolare riferimento non solo all'interazione tra il bambino e la madre (o, comunque, tra il bambino e la figura del suo principale caregiver primario) ma anche e soprattutto a quella tra il paziente e il suo analista (probabilmente a partire dal contributo dei coniugi Baranger, 1961-1962, sino a quelli di vari Autori anche italiani tra i quali si possono citare ad esempio Corrao, Chianese, Riolo, Gaburri e Ferro, 1981-1997).

Ulteriori significative riflessioni al riguardo sono state peraltro sviluppate anche, in questi ultimi due decenni, da vari Autori di estrazione psicologica (anche, ma non solo, psicoanalitica) che hanno cercato una possibile integrazione tra le conoscenze sul ruolo fondamentale che gioca la figura del caregiver nello sviluppo primario del bambino e quelle prodotte da altri settori di ricerca, quali ad esempio le Neuroscienze (Gallese, Migone, Daniel Stern, Morris Eagle, Damasio, Schore, ecc.). Ciò che mi pare opportuno osservare, al riguardo, è ora soprattutto questo:

i) Se, da un lato, appare definitivo l'abbandono del concetto di 'conflitto intrapsichico' a favore di quello di 'conflitto relazionale' almeno da parte alcuni settori della Psicoanalisi Relazionale (e in particolare da parte della Psicoanalisi della Relazione italiana), come si rapporta quest'ultimo concetto di 'conflitto relazionale' con quelli di 'conflitto' in generale e di 'campo', concetti comunque tuttora estremamente presenti e oggetto di vivo interesse da parte della Psicoanalisi italiana ed internazionale?

ii) Se il concetto di 'conflitto relazionale' affonda comunque le sue radici nei lavori degli Psicoanalisti Europei ed Americani che hanno definitivamente abbandonato il modello pulsionale a favore di quello relazionale, come indicato per primo da Ferenczi ormai quasi cent'anni fa, è possibile trovare in qualcuno di tali contributi degli spunti utili per superare l'attuale distanza tuttora presente tra i vari settori della Psicoanalisi che comunque ritengono opportuno, sia pure da punti di vista diversi, continuare ad utilizzarlo sia come modello esplicativo del funzionamento della mente umana che come 'linea d'indirizzo' per le sue applicazioni in psicoterapia?

iii) In quali direzioni si può muovere l'attuale Psicoanalisi, ed in particolar modo la Scuola della Psicoanalisi della Relazione, per produrre le integrazioni necessarie per superare l'attuale divario tuttora rilevante tra Psicoanalisi e Neuroscienze da un lato e Psicoanalisi complessivamente intesa e Psicoanalisi della Relazione dall'altro?

Comincio a tracciare alcune linee generali che spero utili per proseguire la ricerca in tutte queste direzioni, pur nella consapevolezza di non poter 
assolutamente pretendere di disporre al momento attuale di risposte esaustive in alcuno dei suddetti quesiti, in senso inverso a come li ho espressi, nella speranza di poter favorire almeno ulteriori stimoli per la ricerca intorno ad essi oltre a quelli di cui disponiamo sinora. Relativamente, quindi, al terzo di questi quesiti, mi sembra che una spinta generale nelle direzioni da me auspicate possa provenire ad esempio dai contributi di Allan Schore, il quale, da anni, ha indicato nella Teoria dell'Attaccamento di Bowlby un modello potenzialmente utile per superare l'attuale divario tuttora esistente tra Psicoanalisi e Neuroscienze. Come è noto, infatti, tale modello presuppone quattro differenti tipi di attaccamento, che sono specificati nella seguente Figura 6, i quali a loro volta originano quattro differenti stili $d i$ attaccamento ognuno dei quali produce nel bambino l'organizzazione di specifici Modelli Operativi Interni (M.O.I.) che ne costituiranno i patterns fondamentali di funzionamento anche per la vita successiva.

E se tale modello di Bowlby è già stato ampiamente validato ed utilizzato sia da molti psicoanalisti che da molti neuroscienziati, nell'ambito della progressiva integrazione reciproca tra le due discipline da essi rappresentate, il concetto di Modelli Operativi Interni Dissociati (M.O.I.D.) proposto da Albasi in un articolo pubblicato nel 2005 proprio sulla Rivista della S.I.P.Re. 'Ricerca Psicoanalitica' può a mio avviso gettare ulteriori 'ponti' non solo tra la Teoria dell'Attaccamento e la Psicoanalisi della Relazione, ma anche tra quest'ultima e le stesse Neuroscienze, o almeno quella parte di esse che già da anni ha instaurato con la Teoria dell'Attaccamento un confronto reciprocamente costruttivo e produttivo.

Di conseguenza, per quanto riguarda invece il secondo dei tre ampi quesiti poco sopra formulati e proprio sulla base della risposta già appena fornita al terzo di essi, si potrebbe pensare di rispondere positivamente anche ad esso

\begin{tabular}{|c|c|c|c|c|}
\hline Tipo & A & B & C & D \\
\hline Denominazione & Evitante & Sicuro & Ambivalente & Disorganizzato \\
\hline $\begin{array}{l}\text { Stato psichico di base } \\
\text { del bambino }\end{array}$ & Insicuro ed ansioso & $\begin{array}{c}\text { Sufficientemente } \\
\text { tranquillo, - } \\
\text { normale- }\end{array}$ & Ansioso ed insicuro & $\begin{array}{c}\text { Confuso, } \\
\text { estremamente } \\
\text { insicuro ed ansioso }\end{array}$ \\
\hline $\begin{array}{l}\text { Attaccamento } \\
\text { originariamente } \\
\text { stabilito dai genitori } \\
\text { nella loro infanzia } \\
\text { (A.A.I.) }\end{array}$ & $\begin{array}{l}\text { Distanziante } \\
\text { ("Dismissing") }\end{array}$ & $\begin{array}{l}\text { Sufficientemente } \\
\text { sicuro }\end{array}$ & $\begin{array}{l}\text { Insicuro e preoccupato } \\
\text { per il proprio } \\
\text { attaccamento adottato } \\
\text { nella prima infanzia }\end{array}$ & $\begin{array}{l}\text { Traumatico ed } \\
\text { irrisolto } \\
\text { ("Unresolved") }\end{array}$ \\
\hline $\begin{array}{l}\text { Atteggiamento della } \\
\text { madre verso il bambino }\end{array}$ & Rifiutante & $\begin{array}{c}\text { Sensibile ai } \\
\text { bisogni del } \\
\text { bambino }\end{array}$ & $\begin{array}{l}\text { Insensibile ai bisogni } \\
\text { del bambino }\end{array}$ & Caotico, imprevedibile \\
\hline $\begin{array}{c}\text { Atteggiamento del } \\
\text { bambino nella "Strange } \\
\text { Situation" }\end{array}$ & $\begin{array}{c}\text { II bambino non } \\
\text { mostra rabbia verso } \\
\text { la madre nella } \mathbf{S . S .} \text {., } \\
\text { ma la evita quando lei } \\
\text { ritorna }\end{array}$ & $\begin{array}{c}\text { Al ritorno della } \\
\text { madre il bambino } \\
\text { la accoglie, poi } \\
\text { torna a giocare } \\
\text { tranquillo }\end{array}$ & $\begin{array}{c}\text { Ricerca della madre } \\
\text { quando lei è assente, } \\
\text { resistenza rabbiosa } \\
\text { verso di lei al suo } \\
\text { ritorno }\end{array}$ & $\begin{array}{l}\text { II bambino mette in } \\
\text { atto comportamenti } \\
\text { stereotipati, ed è } \\
\text { sorpreso/stupefatto } \\
\text { quando la madre si } \\
\text { allontana. }\end{array}$ \\
\hline $\begin{array}{l}\text { Modelli Operativi Interni } \\
\text { (M.O.L.) del bambino }\end{array}$ & Stile Distanziante & Stile Sicuro & Stile Preoccupato & $\begin{array}{c}\text { Stile } \\
\text { Timoroso-Evitante }\end{array}$ \\
\hline
\end{tabular}

Figura 6. I tipi di attaccamento fondamentali secondo Bowlby (1969-1980) ed i conseguenti stili di attaccamento prodotti nel bambino dai relativi Modelli Operativi Interni (M.O.I.). 
proponendo, come modello di organizzazione mentale su cui si instaura e deve essere gestito il conflitto relazionale di cui vorrei occuparmi ora, un nuovo modello che cerchi di integrare il contributo di Bowlby (che fornisce ampie indicazioni soprattutto riguardo alle modalità di relazione personali verso gli altri) con quello finale sulla struttura mentale proposto da Fairbairn nel 1963, poi modificato da David P. Celani nel volume Fairbairn and the Object Relations Tradition curato da Clarke \& Scharff (2014, pp. 397-429) ed infine da me ulteriormente modificato nel 2015. Di tale modello di organizzazione mentale presento nella seguente Figura 7 una nuova schematizzazione grafica, nella quale i tre 'oggetti interni' sono rappresentati non solo da semplici figure geometriche, ma anche da altrettante immagini fotografiche che cercano di sintetizzarne visivamente le rispettive caratterizzazioni emotive come a mio avviso sono delineate nei lavori dello psicoanalista scozzese.

Partendo pertanto da questo modello modificato di Fairbairn come qui rappresentato, nelle quattro figure seguenti cercherò ora di ipotizzare e conseguentemente rappresentare le differenti situazioni di conflitto relazionale che a mio avviso potrebbero sostanzialmente caratterizzare ciascuno dei quattro stili di attaccamento fondamentali come delineati dall'attuale Teoria dell'attaccamento di Bowlby, utilizzando però soltanto, al riguardo, le reciproche relazioni tra $\mathrm{i}$ tre oggetti interiorizzati come definiti da Fairbairn stesso, e non le strutture psichiche che da essi si sono originate: e questo proprio perché intendo occuparmi di conflitto relazionale e non di conflitto intrapsichico.

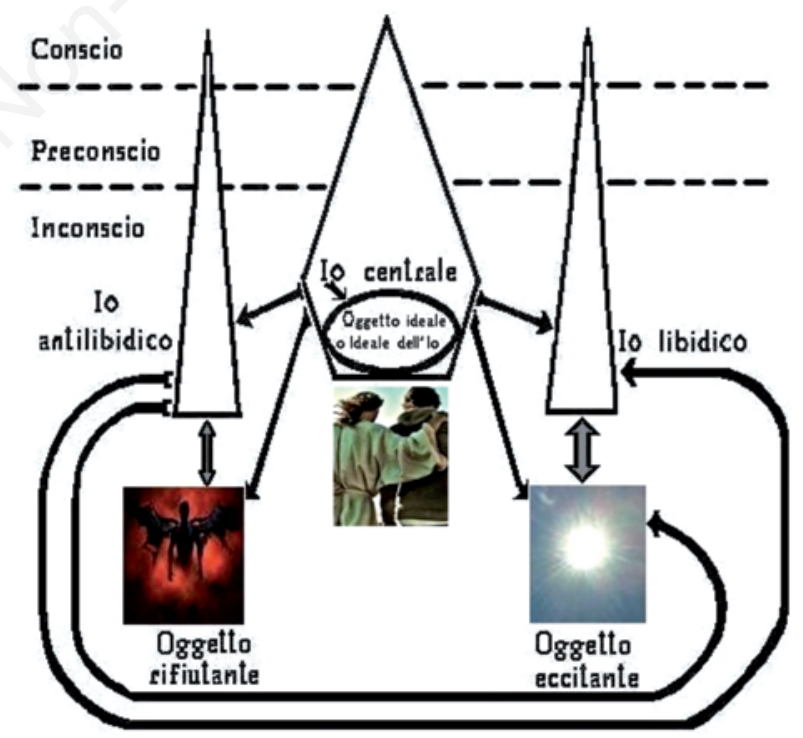

Figura 7. Il modello di Fairbairn del 1963 come modificato da Celani (2014) e Frati (2015). 
In ognuna delle quattro figure seguenti, in particolare, le frecce rosse rappresentano le direzioni in cui ognuno di tali oggetti interiorizzati cerca di esercitare il proprio controllo sugli altre due, o almeno su uno di essi. Naturalmente tali schematizzazioni rappresentano soltanto ipotesi relative a modelli di conflitto relazionale assolutamente teorici, in quanto essi non si presentano mai nella realtà in forma pura ma, nelle singole situazioni cliniche individuali, sono sempre tra di essi combinati nelle più varie forme.

i) Nel modello teorico dello stile di attaccamento sicuro, rappresentato nella Figura 8, l'Oggetto Ideale (o 'Ideale dell'Io') mantiene di solito un sufficiente livello di controllo sia rispetto all'Oggetto interno 'rifiutante' sia rispetto all'Oggetto interno 'eccitante', per cui il conflitto relazionale presentato dalle persone con questo stile di attaccamento risulta di norma abbastanza basso e in genere non particolarmente problematico, salvo particolari situazioni reali di eventi avversi, di stress o di tensioni che possono sempre verificarsi nel corso della vita ma che il più delle volte presentano comunque un carattere transitorio. Buona o comunque generalmente accettabile risulta inoltre, nelle persone caratterizzate principalmente da questo stile di attaccamento, la cosiddetta 'Presenza a sé stessi' (Minolli, 2009).

ii) Nel modello teorico dello stile di attaccamento distanziante, rappresentato nella Figura 9, è invece l'Oggetto interno 'rifiutante' ad esercitare il proprio controllo e dominio sia rispetto all'Oggetto interno 'eccitante' sia verso l'Oggetto Ideale, per cui il conflitto relazionale presentato dalle persone con questo stile di attaccamento risulta di norma abbastanza elevato, con conseguenti comportamenti particolarmente problematici sino a forme autolesive ed anche potenzialmente autodistruttive. Anche la 'Presenza a sé stessi' risulta di norma insufficiente, inadeguata, proprio perché l'Oggetto interno 'rifiutante' tende ad esercitare una funzione di ricorrente, e in taluni casi anche frequente, danneggiamento o comunque turbamento verso l'Oggetto Ideale.

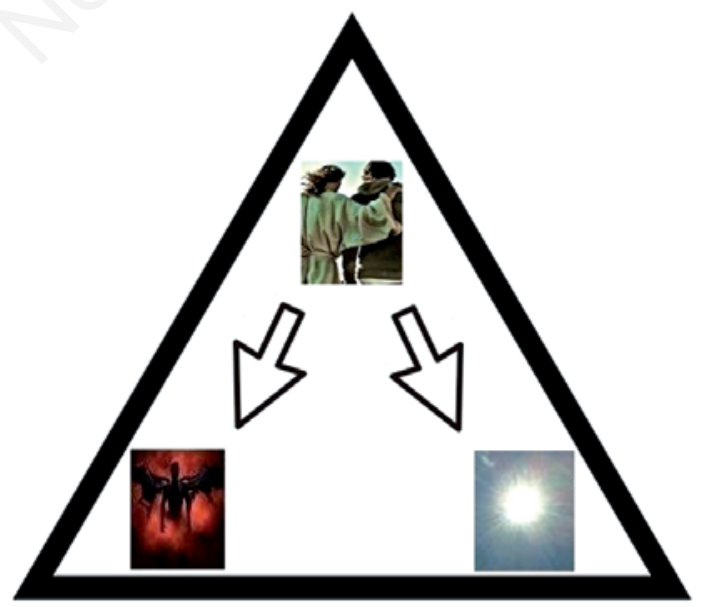

Figura 8. Il modello teorico del conflitto relazionale nelle persone caratterizzate da predominanza di stile di attaccamento sicuro. 
iii) Nel modello teorico dello stile di attaccamento preoccupato, rappresentato nella Figura 10, è invece l'Oggetto interno 'eccitante' ad esercitare il proprio controllo e dominio sia rispetto all'Oggetto interno 'rifiutante' sia verso l'Oggetto Ideale, per cui il conflitto relazionale presentato dalle persone con questo stile di attaccamento risulta di norma abbastanza elevato ma non necessariamente caratterizzato da comportamenti particolarmente problematici sul piano della distruttività etero o autodiretta. Anche la 'Presenza a sé stessi' risulta comunque di norma limitata, perché in questi casi l'Oggetto interno 'eccitante' tende ad esercitare con non trascurabile frequenza una funzione di significativo turbamento verso l'Oggetto Ideale.

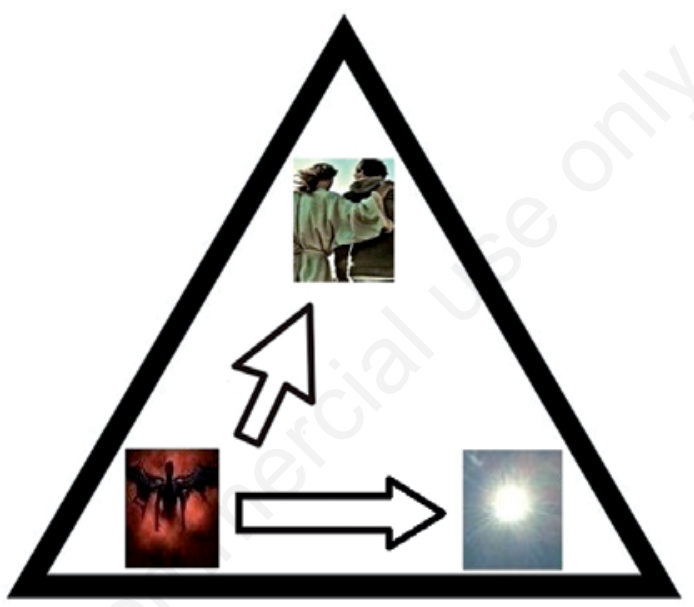

Figura 9. Il modello teorico del conflitto relazionale nelle persone caratterizzate da predominanza di stile di attaccamento distanziante.

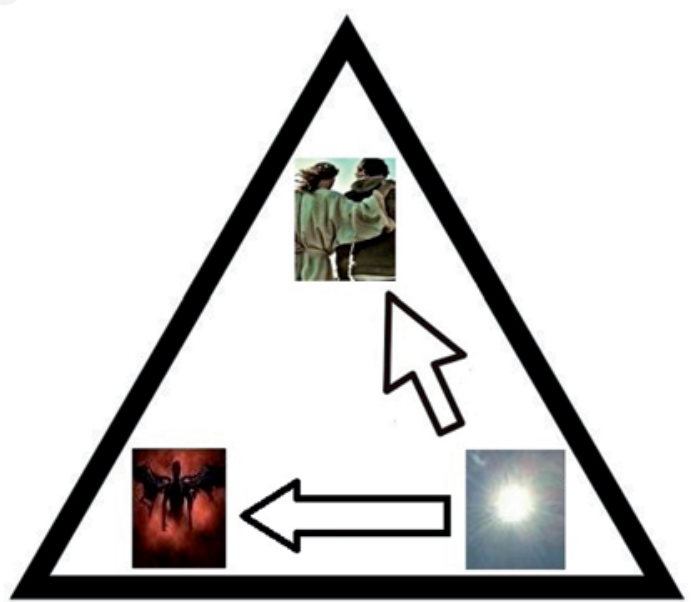

Figura 10. Il modello teorico del conflitto relazionale nelle persone caratterizzate da predominanza di stile di attaccamento preoccupato. 
iv) Nella Figura 11, infine, è rappresentato il modello teorico dello stile di attaccamento timoroso-evitante, nel quale sia l'Oggetto interno eccitante sia l'Oggetto interno rifiutante tendono ad esercitare il proprio controllo e dominio verso l'Oggetto Ideale, per cui il conflitto relazionale presentato dalle persone con questo stile di attaccamento risulta di norma abbastanza elevato e potenzialmente caratterizzato da comportamenti particolarmente problematici sia sul piano della distruttività etero o autodiretta sia, in forma anche superiore, da ambivalenza emotiva ed affettiva. Anche la 'Presenza a sé stessi' risulta di norma scarsa o comunque significativamente limitata, perché in questi casi sono sia l'Oggetto interno 'rifiutante' sia l'Oggetto interno 'eccitante' che tendono ad esercitare con elevata frequenza una non trascurabile funzione di significativo turbamento verso l'Oggetto Ideale.

La gravità di ognuna di queste forme di conflitto relazionale, ovviamente, non è comunque determinata soltanto dal tipo di controllo che uno o due oggetti interni tendono ad esercitare verso gli altri, ma anche dal livello quantitativo con cui tale controllo viene esercitato.

Pertanto, dopo aver potuto rispondere - attraverso l'utilizzo di una sorta di 'modello del conflitto relazionale interno' fondato su una proposta di integrazione dei contributi teorici di Bowlby e di Fairbairn - in modo sostanzialmente affermativo al secondo dei tre quesiti sopra espressi, cercherò ora di fornire una risposta anche al primo di essi. Per far ciò utilizzerò principalmente i contributi teorici rispettivamente forniti da un recente strumento diagnostico di stampo psicoanalitico, l'OPD (2009), sulla base dell'analisi che ne hanno fatto alcuni autori della scuola della psicoanalisi della relazione ed in particolare Maria Luisa Tricoli (2017).

Rileva infatti questa autrice che l'OPD, 'aderendo ad una visione conflittuale, ma non strettamente freudiana, del funzionamento umano, indivi-

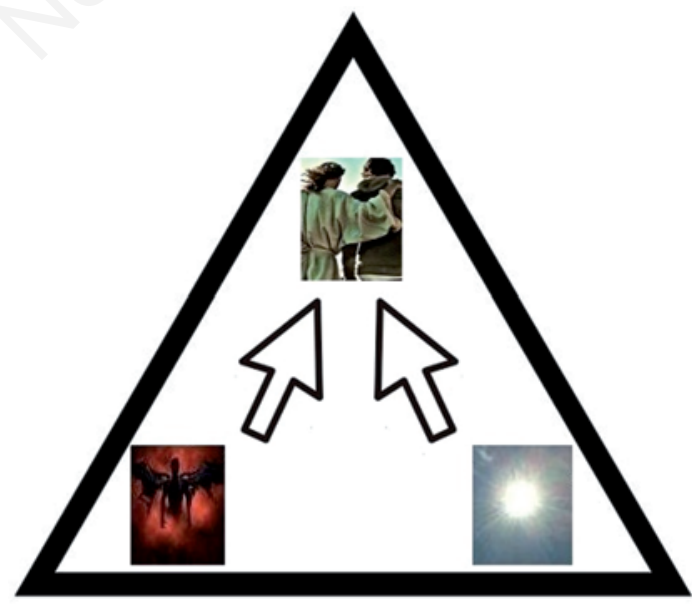

Figura 11. Il modello teorico del conflitto relazionale nelle persone caratterizzate da predominanza di stile di attaccamento timoroso-evitante. 
dua sette tipi di conflitto, cioè di tensioni irrisolte che determinano il vissuto e il comportamento della persona e che possono essere inferite dal colloquio clinico (Stanghellini 2009, pp. 215-216): dipendenza vs autonomia, sottomissione vs controllo; accudimento vs autarchia; valorizzazione del sé vs valorizzazione dell'altro; tendenze egoistiche $v s$ tendenze pro-sociali; conflitti edipico-sessuali; conflitti relativi all'identità che originano da rappresentazioni contraddittorie del Sé' (Tricoli, 2017).

Osserva, al riguardo, la medesima Autrice: 'Tralasciando il fatto che l'OPD, conformandosi ad un preciso modello teorico, è interessato all'aspetto conflittuale che sta a monte delle manifestazioni osservabili, le contrapposizioni individuate, trascurando le ultime due voci dell'elenco, possono essere considerate attitudini proprie di ogni essere umano che investono unitariamente l'area degli affetti, del pensiero e delle manifestazioni comportamentali' (Idem, p. 132).

Alla luce di tutte queste considerazioni mi sembra pertanto possibile concludere questa mia disamina del concetto di 'conflitto relazionale', anche al fine di dare finalmente una risposta anche al primo dei tre quesiti sopra formulati, evidenziando come, nel panorama complessivo della Psicoanalisi italiana ed internazionale contemporanea, non solo esso appaia tuttora strettamente connesso sia a quello di 'conflitto' in generale che a quello di 'campo' (inteso proprio come 'campo relazionale'), ma anche - e forse soprattutto che proprio attraverso l'analisi congiunta e comune di entrambi questi aspetti così inestricabilmente presenti ed interagenti nella vita di ogni persona - cioè, appunto, il 'conflitto' ed il 'campo' propri di quella persona che a noi si rivolge - possa a volte rendersi per lei possibile, in ambito clinico e psicoterapeutico, una sufficientemente adeguata comprensione dei suoi bisogni, delle sue difficoltà, ma anche delle sue potenzialità.

$\mathrm{O}$ anche, detta in altri termini, che l'accompagnamento di ciascuno dei nostri pazienti verso l'obiettivo di un più soddisfacente benessere psicologico, di una migliore capacità di gestione dei propri conflitti, di una maggiore padronanza della propria vita (e cioè, per riprendere il fondamentale concetto insegnatoci da Michele Minolli, di una migliore 'Presenza a se stessi'), debba necessariamente prevedere, per noi, l'acquisizione ed il riconoscimento di un significato e di un senso all'interno del campo relazionale di quel paziente, dei suoi affetti e pensieri, dei suoi comportamenti, dei suoi stessi conflitti passati ed attuali. 


\section{BIBLIOGRAFIA}

Albasi, C. (2005). Modelli operativi interni dissociati, ovvero che cosa succede quando non viene riconosciuta la specificità. Ricerca Psicoanalitica, 16, 3: 331-354.

Aron, L. (1996). Menti che si incontrano. Milano: Raffaello Cortina Editore, 2004.

Aron, L. \& Sommer Anderson, F. (edited by) (1998). Il corpo nella prospettiva relazionale. Roma: La Biblioteca, 2004.

Bacciagaluppi, M. (1996). Le idee di Erich Fromm sulla 'tecnica' psicoanalitica. Psicoterapia e scienza umane, 23(3), 1989.

Bacciagaluppi, M. (2012). Paradigmi in psicoanalisi. Un modello integrato. Pisa: Edizioni ETS, 2012.

Baranger, W. M. (1961-2). La situazione analitica come campo bipersonale. Milano: Cortina, 1990.

Bowlby, J. (1969). Attaccamento e perdita. Vol. 1. L'attaccamento alla madre. Torino: Boringhieri, 1989.

Bowlby, J. (1973). Attaccamento e perdita. Vol. 2. La separazione dalla madre. Torino: Bollati Boringhieri, 1978.

Brenner, C. (1955). Breve corso di psicoanalisi. Firenze: Martinelli, 1976.

Brenner, C. (1976). Tecnica psicoanalitica e conflitto psichico. Firenze: Martinelli, 1978.

Bromberg, M.B. (1998/2001). Clinica del trauma e della dissociazione. Milano: Raffaello Cortina Editore, 2007.

Celani, D.P. (1994). The illusion of love: why the battered woman returns to her abuser. New York: Columbia University Press.

Celani D.P. (2010). Fairbairn's Object Relations Theory in the Clinical Setting. New York: Columbia University Press.

Celani, D.P. (2014). Revising Fairbairn's structural theory. In: Clarke \& Scharff, 2014, pp. 397-429.

Clarke, G. \& Scharff D. (2014). Fairbairn and the Object Relations Tradition. London: Karnac. 2014.

Fairbairn, W.R.D. (1940). Schizoid Factors in the Personality. Ital. trans.: Fattori schizoidi nella personalità, in Studi psicoanalitici sulla Personalità, cit., pages 25-51.

Fairbairn, W.R.D. (1941). Riesame della psicopatologia delle psicosi e delle psiconevrosi. In: Fairbairn. W.D.R. Studi psicoanalitici sulla personalità. Torino: Bollati Boringhieri, 1970.

Fairbairn, W.R.D. (1943). The Repression and the Return of Bad Objects (with Special Reference to the 'War Neuroses'). Ital. trans.: La rimozione e il ritorno degli oggetti cattivi, in Studi psicoanalitici sulla Personalità, cit., pp. 85-109.

Fairbairn, W.R.D. (1944). Endopsychic Structure considered in Terms of Object.Relationships. Ital. trans.: La struttura endopsichica considerata in termini di relazioni oggettuali, in Studi psicoanalitici sulla Personalità, cit., pp. 110-168.

Fairbairn, W.R.D. (1946). Object-Relationships and Dynamic Structure. Ital. trans.: Relazioni oggettuali e struttura dinamica, in Studi psicoanalitici sulla Personalità, cit., pp. 169-184.

Fairbairn WRD (1952). Psychoanalytic studies of the personality. Ital. trans. Studi psicoanalitici sulla personalità. Torino: Bollati Boringhieri, 1970.

Fairbairn, W.R.D. (1958). On the nature and aims of psychoanalytic treatment. Int. J. Psychoanal., Vol. XXXIV, 1958, pp. 374-385. Trad. it.: Natura e scopi del trattamento psicoanalitico, in Il piacere e l'oggetto: scritti 1952-1963, cit., pp. 143-168.

Fairbairn, W.R.D. (1952-1963). Il piacere e l'oggetto: scritti 1952-1963. Edited by Franco Orsucci (Psiche e coscienza). Roma: Astrolabio Ubaldini, 1992.

Fairbairn, W.R.D. (1963). Synopsis of an Object-Relations Theory of Personality. Int. J. Psychoanal., Vol. XLIV, 1963, pag. 224. Ital. trans.: Sinossi della teoria delle relazioni 
oggettuali applicata alla personalità. In Il piacere e l'oggetto: scritti 1952-1963, cit., pp. $169-170$

Ferenczi, S. (1924). Thalassa. Saggio sulla teoria della genitalità. Milano: Cortina, 1965.

Ferenczi, S. (1920-32). Frammenti e annotazioni. In Opere, vol. 4. Milano: Raffaello Cortina, 2002.

Ferenczi, S. (1932a). La confusione delle lingue tra adulti e bambini. In Opere, vol. 4. Milano: Raffaello Cortina, 2002.

Ferenczi, S. (1932b). Diario clinico. Milano: Raffaello Cortina, 1988.

Fontana, M. (2016). La psicopatologia in Psicoanalisi della Relazione. Retrieved from: https://sipreonline.it/wp-content/uploads/2016/10/Fontana_Psicopatol_La-psicopat-inPsi-Rel.pdf

Fontana, M. (2017). La diagnosi e le sue implicazioni nella clinica psicoanalitica. Roma: Giovanni Fioriti Editore, 2017.

Frati, F. (2012). Il lato oscuro della mente. L'io di fronte ai cambiamenti. Molfetta: Edizioni La Meridiana, 2012.

Frati, F. (2013). La rilevanza dell'opera di W. R. D. Fairbairn per la metapsicologia e la psicoterapia contemporanee. Ricerca Psicoanalitica: Anno XXIV, n.2/2013, pp. 105-125. Milano: Franco Angeli, 2013.

Frati, F. (2015). Il problema della 'rimozione dell'oggetto buono' nel modello teorico-clinico di Fairbairn. Psicoterapia e Scienze Umane: n.3, Vol. XLIX. Milano: Franco Angeli, 2015.

Freud, A. (1936). L'Io e i meccanismi di difesa. Firenze: Martinelli, 1967.

Freud, S., \& Breuer, J. (1892-1895). Studi sull'isteria. Opere, vol. 1: 161-439. Torino: Boringhieri, 1976.

Freud, S. (1894), Le neuropsicosi da difesa. Opere, vol. 2: 121-134. Torino: Boringhieri, 1976. Freud, S. (1895). Progetto di una Psicologia. Opere, vol. 2: 201-284. Torino: Boringhieri, 1976. Freud, S. (1899). Ricordi di copertura. Opere, vol. 2: 435-453. Torino: Boringhieri, 1976. Freud, S. (1899 [1900]). L'interpretazione dei sogni. Opere, vol. 3: 3-565. Torino: Boringhieri, 1966.

Freud, S. (1904). Psicopatologia della vita quotidiana. Opere, vol. 4: 57 - 298. Torino: Boringhieri, 1970.

Freud, S. (1915-17). Introduzione alla psicoanalisi. Opere, vol. 8: 191-613. Torino: Boringhieri, 1976.

Freud, S. (1915-17 e 1932). Introduzione alla psicoanalisi. Prima e seconda serie di lezioni. Torino: Boringhieri, 1969.

Freud, S. (1917a). Supplemento metapsicologico alla teoria del sogno. Opere, vol. 8: 89101. Torino: Boringhieri, 1976.

Freud, S. (1917b). Lutto e melanconia. Opere, vol. 8: 102-118. Torino: Boringhieri, 1976.

Freud, S. (1920). Al di là del principio di piacere. Opere, vol. 9: 187-249. Torino: Boringhieri, 1976.

Freud, S. (1922a). Due voci di enciclopedia: 'Psicoanalisi'e 'Teoria della Libido'. Opere, vol. 9: 435-462. Torino: Boringhieri, 1976

Freud, S. (1922b). L'Io e l'Es. Opere, vol. 9: 469-520. Torino: Boringhieri, 1976.

Freud, S. (1923). Nevrosi e psicosi. Opere, vol. 9: 607-615. Torino: Boringhieri, 1976.

Freud, S. (1926). Inibizione, sintomo e angoscia. Opere, vol. 10: 231-317. Torino: Boringhieri, 1976.

Freud, S. (1932). Introduzione alla psicoanalisi (nuova serie di lezioni). Opere, vol. 11: 115284. Torino: Boringhieri, 1979.

Freud, S. (1938 [1940]). Compendio di psicoanalisi. Opere, vol. 11: 569-634. Torino: Boringhieri, 1979.

Greenberg, J., \& Mitchell, S.A. (1983). Object relations in psychoanalytic theory. Ital. trans. Le relazioni oggettuali nella teoria psicoanalitica. Bologna: Il Mulino, 1986. 
Gruppo di lavoro OPD (a cura di A. De Coro) (2002). Diagnosi psicodinamica operazionalizzata. Presupposti teorici e applicazioni cliniche. Milano: Masson, 2002.

Guntrip, H. (1961). Struttura della personalità e interazione umana. Torino: Boringhieri, 1971.

Hinshelwood, R.D. (1989). A dictionary of Kleinian thought. Ital. trans. Dizionario di psicoanalisi kleiniana. Milano: Raffaello Cortina, 1990.

Hirsch, I. (2008). Coasting in the countertransference. Conflicts of Self Interest between Analyst and Patient. New York: The Analytic Press, 2008.

Holt, R.R. (1989). Freud reappraised. Ital. trans. Ripensare Freud. Torino: Bollati Boringhieri, 1994.

Klein, M. (1929). La personificazione nel gioco infantile. In Scritti: 1921-1958. Torino: Bollati Boringhieri, 1978, pp. 227-238.

Klein, M. (1932). La psicoanalisi dei bambini. Firenze: Martinelli, 1970.

Klein, M. (1957). Invidia e gratitudine. Firenze: Martinelli, 1985.

Klein, M. (1958). Scritti: 1921-1958. Torino: Bollati Boringhieri, 1978.

Klein, M. \& Riviere, J. (1964). Amore, odio e riparazione. Roma: Astrolabio, 1969.

Kohut, H. (1971). The analysis of the self. Ital. trans.: Narcisismo e analisi del Sé. Torino: Boringhieri, 1976.

Laplanche, J., \& Pontalis, J.B. (1967). Vocabulaire de la psychanalyse. Ital. trans. Enciclopedia della Psicoanalisi. Bari: Laterza, 1973.

Liotti, G. (1999). Disorganization of attachment as a model for understanding dissociative psychopathology. In: Solomon, J., George, C. (edited by). Attachment disorganization. New York: Guilford Press, 1999.

Liotti, G., \& Farina, B. (2011). Sviluppi traumatici. Milano: Raffaello Cortina Editore, 2011.

Minolli, M. (1996). La psicoanalisi della relazione. Ricerca Psicoanalitica, VII(1-2), 117-133.

Minolli, M. (1998). 1897-1997 Cento anni senza trauma reale. Intervista con Marco Bacciagaluppi. Ricerca Psicoanalitica, IX(1), 59-69.

Minolli, M. (2004). Per un Io-Soggetto fatto di legami. Ricerca Psicoanalitica, XV(3), 317-329.

Minolli, M. (2005). Psicoanalisi della Relazione di coppia. Ricerca psicoanalitica, XVI (3), 219-242.

Minolli, M. (2005). Per un Io-soggetto come sistema. Ricerca psicoanalitica, XVI(3) 355-374.

Minolli, M., \& Coin, R. (2006). Per una psicoanalisi della relazione. Psicoterapia e Scienze Umane: Anno XL, 3, 641-652. Milano: Franco Angeli, 2006.

Minolli, M., \& Coin, R. (2007). Amarsi, amando. Per una psicoanalisi della relazione di coppia. Milano: Franco Angeli.

Minolli, M. (2009). Psicoanalisi della relazione. Milano: Franco Angeli.

Minolli, M. (2011). Tu sei me e io sono te. Ricerca Psicoanalitica, XXII(2).

Minolli, M. (2015). Essere e divenire. La sofferenza dell'individualismo. Milano: Franco Angeli. 2015,

Mitchell, S.A. (1988). Relational concepts in psychoanalysis: an integration. Ital. trans. Gli orientamenti relazionali in psicoanalisi. Torino: Bollati Boringhieri, 1993.

Mitchell, S.A. (1993). Speranza e timore in psicoanalisi. Torino: Bollati Boringhieri, 1995.

Mitchell, S.A. (1997). Psychoanalysis and the degradation of romance. Psychoanalytic Dialogues 7, 23-42.

Mitchell, S.A., \& Aron, L, (a cura di) (1999). Relational psychoanalysis. The emergence of a tradition. Hillsdale, NJ: The Analytic Press.

Mitchell, S.A. (2000). Il modello relazionale, dall'attaccamento all'intersoggettività. Milano: Raffaello Cortina, 2002.

Mitchell, S. (2001). Interview with... (AA.VV). Ricerca Psicoanalitica, XII(1), 32.

Morin, E. (1981). Computo ergo sum. Ricerca Psicoanalitica, XVIII(3), 263-282.

Morin, E. (1985). Le vie della complessità. In Bocchi, G., Ceruti, M. (edited by) (1985). La sfida della complessità. Milano: Feltrinelli, 1985. 
Rugi, G. (1998). Riflessioni sul modello psicoanalitico di campo. Retrieved from:

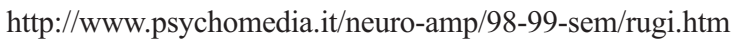

Schore, A.N. (2003). La regolazione degli affetti e la riparazione del sé. Roma: Astrolabio, 2008.

Skolnick, N.J. \& Warshaw, S.C. (edited by) (1992). Relational perspectives in psychoanalysis. Hillsdale, NJ: The Analytic Press.

Stanghellini, G. \& Rossi Monti, M. (edited by) (2009). Psicologia del patologico. Una prospettiva fenomenologico-dinamica. Milano: Cortina, 2009.

Stanghellini, G. (2009). Conflitto. In: Stanghellini, G. \& Rossi Monti, M. (edited by), Psicologia del patologico. Una prospettiva fenomenologico-dinamica. Milano: Cortina, 2009.

Stern, D.B. (1997). Unformulated experience from dissociation to imagination in psychoanalysis. Hillsdale, NJ: The Analytic Press, 1997.

Stern, D.B. (2010). Partners in thought. working with unformulated experience, dissociation, and enactment. New York: Routledge, 2010.

Stern, D.N. (1985). Il mondo relazionale del bambino. Torino: Bollati Boringhieri, 1987.

Stern, D.N., Sander, L., \& Nahum, J., Harrison, A.M., Lyons-Ruth, K., Morgan, A.C., Bruschweilerstern, N. \& Tronick, E.Z. (1998). Non-interpretative mechanisms in psychoanalytic therapy: the 'something more' than interpretation. International Journal of Psychoanalysis, 79, 903-921.

Stern, D.N. (2002). Why do people change in psychotherapy? Continuing Education Seminar, ceseminars@aol.com, Los Angeles, CA.

Stern, D.N. (2004). Il momento presente. Milano: Raffaello Cortina Editore, 2003.

Sullivan, C.T. (1962). Freud and Fairbairn: two Theories of Ego Psychology. Doleystown Foundation, PA, 1963.

Sullivan, H.S. (1959). The Interpersonal Theory of Psychiatry. New York, Norton, 1959. (Ital. trans.: Teoria interpersonale della Psichiatria. Milano: Feltrinelli, 1962).

Sutherland, J.D. (1961). Introduction. In Guntrip, H., 1961, cit.

Sutherland, J.D. (1963). Object-relations theory and the conceptual model of psychoanalysis. British Journal of Medical Psychology, 36, 109-124.

Sutherland, J.D. (1980). The British object relations theorists: Balint, Winnicott, Fairbairn, Guntrip. Journal of American Psychoanalytic Association, 28, 829-860.

Sutherland, J.D. (1989). Fairbairn's Journey into the Interior. London: Free Association Books, 1989.

Sutherland, J.D. (1994). The Autonomus Self(J.S. Sharff, Ed.). Northvale, NJ: Jason Aronson.

Suttie, I.D. (1935). Le origini dell'amore e dell'odio. Prefazione di John Bowlby. Introduzione di Dorothy Heard. Prefazione alla prima edizione di James A. Hadfield. Postfazione di Franco Borgogno. Trans. by Francesco Capello. Torino: Centro Scientifico Editore, 2007.

Task Force OPD (2009). OPD-2. Diagnosi psicodinamica operazionalizzata. Manuale per la diagnosi e la pianificazione del trattamento. Trad, it. Milano: FrancoAngeli, 2009.

Tricoli, M.L. (2001). Dal controtransfert alla self-disclosure: la scoperta della soggettività dell'analista. Ricerca Psicoanalitica, 12(3), 229-245.

Tricoli, M.L. (2017). La diagnosi: un processo di senso. In: Fontana, M. (2017). La diagnosi e le sue implicazioni nella clinica psicoanalitica (pp. 127-143). Roma: Giovanni Fioriti Editore.

Winnicott, D.W. (1958). La capacità di essere solo. Ital. trans. in: Winnicott, D. W. (1965). Sviluppo affettivo e ambiente. Studi sulla teoria dello sviluppo affettivo. Roma: A. Armando, 1974.

Winnicott, D.W. (1965). The Maturational Process and the Facilitating Environment (1957-1963). New York: International Universities Press, 1965. Trad. it.: Sviluppo 
affettivo e ambiente. Studi sulla teoria dello sviluppo affettivo. Roma: A. Armando, 1970.

Winnicott, D.W. (1971). Gioco e realtà. Roma: A. Armando, 1974.

Zito, S. (2017). Diagnosi e 'specificità' della persona. In: Fontana, M. (2017). La diagnosi e le sue implicazioni nella clinica psicoanalitica (pp. 145-158). Roma: Giovanni Fioriti Editore.

Conflitto di interessi: l'autore dichiara che non vi sono potenziali conflitti di interessi.

Approvazione etica e consenso a partecipare: non necessario.

Ricevuto per la pubblicazione: 8 marzo 2021.

Accettato per la pubblicazione: 8 settembre 2021.

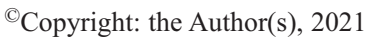

Licensee PAGEPress, Italy

Ricerca Psicoanalitica 2021; XXXII:535

doi:10.4081/rp.2021.535

This article is distributed under the terms of the Creative Commons Attribution Noncommercial License (by-nc 4.0) which permits any noncommercial use, distribution, and reproduction in any medium, provided the original author(s) and source are credited. 